Article

\title{
How Is Urban Greenness Spatially Associated with Dockless Bike Sharing Usage on Weekdays, Weekends, and Holidays?
}

\author{
Feng Gao ${ }^{1,2,3}$, Shaoying Li ${ }^{1}{ }^{*}$, Zhangzhi Tan ${ }^{4,5}$, Xiaoming Zhang ${ }^{2,3}$, Zhipeng Lai ${ }^{1}$ and Ziling Tan ${ }^{1}$ \\ 1 School of Geography and Remote Sensing, Guangzhou University, Guangzhou 510006, China; \\ 2111801048@gzhu.edu.cn (F.G.); 2112001055@e.gzhu.edu.cn (Z.L.); 2112001077@e.gzhu.edu.cn (Z.T.) \\ 2 Guangzhou Urban Planning and Design Survey Research Institute, Guangzhou 510060, China; \\ zhangxiaoming@gzpi.com.cn \\ 3 Guangdong Enterprise Key Laboratory for Urban Sensing, Monitoring and Early Warning, \\ Guangzhou 510030, China \\ 4 School of Intelligent Systems Engineering, Sun Yat-sen University, Guangzhou 510085, China; gistop@126.com \\ 5 Shenzhen Urban Transport Planning Center Co., Ltd., Shenzhen 518057, China \\ * Correspondence: lsy@gzhu.edu.cn
}

Citation: Gao, F.; Li, S.; Tan, Z.; Zhang, X.; Lai, Z.; Tan, Z. How Is Urban Greenness Spatially Associated with Dockless Bike Sharing Usage on Weekdays, Weekends, and Holidays? ISPRS Int. J. Geo-Inf. 2021, 10, 238 https://doi.org/10.3390/ijgi10040238

Academic Editors: Wolfgang Kainz and Giuseppe Borruso

Received: 24 February 2021

Accepted: 4 April 2021

Published: 7 April 2021

Publisher's Note: MDPI stays neutra with regard to jurisdictional claims in published maps and institutional affiliations.

Copyright: (C) 2021 by the authors Licensee MDPI, Basel, Switzerland. This article is an open access article distributed under the terms and conditions of the Creative Commons Attribution (CC BY) license (https:// creativecommons.org/licenses/by/ $4.0 /)$.

\begin{abstract}
Dockless bike sharing plays an important role in residents' daily travel, traffic congestion, and air pollution. Recently, urban greenness has been proven to be associated with bike sharing usage around metro stations using a global model. However, their spatial associations and bike sharing usage on public holidays have seldom been explored in previous studies. In this study, urban greenness was obtained objectively using eye-level greenness with street-view images by deep learning segmentation and overhead view greenness from the normalized difference vegetation index (NDVI). Geographically weighted regression (GWR) was applied to fill the research gap by exploring the spatially varying association between dockless bike sharing usage on weekdays, weekends, and holidays, and urban greenness indicators as well as other built environment factors. The results showed that eye-level greenness was positively associated with bike sharing usage on weekdays, weekends, and holidays. Overhead-view greenness was found to be negatively related to bike usage on weekends and holidays, and insignificant on weekdays. Therefore, to promote bike sharing usage and build a cycling-friendly environment, the study suggests that the relevant urban planner should pay more attention to eye-level greenness exposure along secondary roads rather than the NDVI. Most importantly, planning implications varying across the study area during different days were proposed based on GWR results. For example, the improvement of eye-level greenness might effectively promote bike usage in northeastern and southern Futian districts and western Nanshan on weekdays. It also helps promote bike usage in Futian and Luohu districts on weekends, and in southern Futian and southeastern Nanshan districts on holidays.
\end{abstract}

Keywords: dockless bike sharing; cycling; urban greenness; built environment; street view image data; metro station

\section{Introduction}

Free-floating dockless bike sharing is a low-carbon, convenient, flexible, and efficient travel mode, which has been rapidly popularized and developed in many cities in China. The rise of dockless bike sharing has changed the daily travel mode of residents, solving the "last mile" travel problem in rail transit station connections [1-5]. Moreover, cycling is beneficial to both physical health and the environment [3-7]. On the one hand, physical activities such as cycling can help improve health both physically and mentally by reducing the rate of diseases including cardiovascular diseases [8], obesity [9], diabetes, and some types of cancer $[10,11]$. Cycling for leisure or commuting purposes may increase the level and duration of moderate to intense physical activities of residents given that it is both low-cost and convenient, and can be integrated into residents' daily lives [6,12]. On the 
other hand, dockless bike sharing in metropolis contributes to easing traffic congestion, thereby reducing air and noise pollution and $\mathrm{CO}_{2}$ emissions [13,14].

Exploring the association between dockless bike sharing usage and urban greenness is of great importance in many ways, including better understanding of the relationship between bike usage and urban greenness, urban planning and design for cycling-friendly environments, and the encouragement of bike sharing usage [3-5]. Although many previous studies have attempted to analyze the relationship between urban greenness and dockless bike sharing usage with global regression models, few have explored the spatial heterogeneity of the association between urban greenness and bike usage [3-5]. Previous studies with global models are insufficient to understand the bike-greenness relationship for the following reasons. First, the results of global models, including multilevel logistic regression [3], multivariate Poisson regression [4], and multiple linear regression [5], in related studies can only generate global results; that is, the variable coefficient is global and identical across the study area, and is incapable of reflecting their spatial associations. Furthermore, related planning suggestions and policymaking in previous studies might lack focus without considering spatial heterogeneity. For instance, some studies have suggested that eye-level greenness is positively related to cycling and, hence, should be improved. However, the differences in the spatial variation of the association between greenness and cycling in the study area and how to promote greenness planning for different regions in the study area remain unclear and unsolved in previous studies. In addition, although bike sharing usage on weekdays and weekends has been explored in association with urban greenness, cycling during public holidays is also a non-negligible research object, and was seldom involved in previous studies.

The aim of this article is to fill the above two research gaps by applying a geographically weighted regression (GWR) model and obtaining bike sharing usage including weekdays, weekends, and holidays to address the following research questions:

- How are urban greenness indicators spatially associated with dockless bike sharing usage?

- How different are the associations between greenness and bike sharing usage on weekdays, weekends, and holidays?

To answer these questions, first, two urban greenness indicators, eye-level greenness and overhead view greenness, were measured with street view images using the deep learning segmentation method and Landsat8 images, respectively. Then, the integration of backward stepwise regression and GWR was applied to quantify the spatial association between urban greenness and dockless bike sharing usage. The backward stepwise linear regression was used to solve the multi-collinearity issue and select significant variables. The benefit of applying the GWR model is that it can obtain the local coefficients of influencing factors on bike sharing usage. Thus, urban planning suggestions for different regions can be proposed based on GWR results. The results are helpful for urban planners to improve urban greenness to promote dockless bike sharing usage efficiently, considering spatial heterogeneity. This study consisted of five parts. Section 2 presents the literature review. Section 3 introduces the data source, study area, and methods involved. Section 4 presents the results and discussions of the study, including eye-level urban greenness extraction, spatial distribution of dockless bike sharing usage, stepwise linear regression and variable selection, and spatial variation of coefficients from GWR models. Section 5 concludes the study results, makes proposals based on the results, and presents the prospects for future research.

\section{Related Works}

Many studies have been conducted on both cycling behavior and urban greenness, as well as the relationship between them, which are listed in Table 1. First, the influential mechanism of bike-sharing usage or cycling frequency is the focus of bike-related studies with spatial analysis methods. For example, Shen et al. systematically quantified the impacts of built environment factors on dockless bike-sharing usage in Singapore using a 
spatial autoregression model and found that land use mixture, public transit, and cycling facilities were positively related to bike usage. Gao et al. further explored the modifiable areal unit problem in the modeling procedure of the influential mechanism of bike-sharing usage in Shenzhen using a geographical detector model [2]. Nickkar et al. found that land use of origin, destination, and gender of users were associated with bike usage in Baltimore with multinomial logistic regression. Among previous studies, dockless bikesharing usage can be categorized into two modes: cycling as the sole travel mode and the metro-bike transfer mode [4,15-18]. Among them, the metro-bike transfer mode has received great interest from both researchers and urban planners owing to its remarkable advantages such as reducing private vehicle use [17], enhancing public transit usage, and promoting travel efficiency and convenience [18]. Therefore, exploring the impacts of driving factors on dockless bike-sharing usage in the metro-bike transfer mode remains the focus of cycling-related studies $[2,4]$, which can shed light on urban planning and design to provide a cycling-friendly built environment for the purpose of encouraging such an environmentally friendly travel mode and residents' physical condition [19-22].

In terms of the influential mechanism of bike usage in previous studies, urban greenness has been proven to be an essential built environment factor affecting the usage of dockless bike sharing because it can attract cycling activities both emotionally and aesthetically [23-25]. Although the definition of urban greenness in related studies varies according to different research fields and subjects, it is usually defined as vegetation, trees, and grass of parks, squares, open green space, and along roads [3,26]. However, previous studies of the relationship between urban greenness and cycling are inconsistent [13,16,19,27-31] Some studies found that urban greenness is positively associated with cycling activities. For example, Krenn et al. (2014) found that street greenness level is positively related to the preference of cyclists' route choices [19]. Porter et al. (2019) proved that tree canopy coverage and parks positively affect the cycling frequency in four cities in the United States [31]. Cole-Hunter et al. (2015) found that greenness is a positive influencing factor on the willingness of cycling commuting travel around the workplace [28]. Fraser and Lock (2011) concluded that urban greenness is weakly associated with cycling by reviewing 21 relevant studies [13]. Ruoyu et al. (2020) found that eye-level greenness was positively associated with cycling frequency on weekdays and on weekends within three buffers around metro stations (500 m, $1000 \mathrm{~m}$, and $1500 \mathrm{~m}$ ) [4]. However, no association [24,32] and negative association $[20,21]$ were found in some cases. For instance, Christiansen et al. (2016) found that urban greenness was not significantly associated with cycling frequency based on a comparative study among fourteen cities globally [24]. Sun et al. (2017) claimed that no association was observed between cycling and participants' proximity to green spaces [32]. In addition, Mertens et al. (2017) found that the number of trees was negatively associated with cycling duration in five cities [20,21].

The previously noted inconsistency might be partly attributed to the difference in urban greenness estimation methods [13,16,19,27-31]. Traditional urban greenness estimation methods, such as questionnaires [33] and field investigation methods, are costly and inefficient [34]. Recently, Yi et al. (2019) and Yiyong et al. (2020) uncovered the inconsistency by proving that eye-level greenness extracted from street view image data with deep learning methods was positively associated with cycling activities and dockless bike-sharing usage, respectively, rather than overhead view greenness measured by the normalized difference vegetation index (NDVI). Additionally, some studies have shown that eye-level greenness might better measure greenness out of cyclists' subjective feelings and sights than overhead view greenness [3-5,30,35-38]. Hence, street view image data and deep learning segmentation methods have become efficient data sources and assessment methods for measuring urban greenness, which has been applied in transport geography, psychology, and crime studies $[3,35,37,38]$.

Several gaps need to be filled in studies of association between urban greenness and dockless bike sharing usage. Firstly, previous studies compared the association on weekdays and weekend, although without considering the bike usage on holidays such 
as national holidays of China [4,5]. Secondly, spatial models have never been applied in studies of association between urban greenness and dockless bike sharing usage, hence resulting in the lack of the exploration of the spatially varying impacts of urban greenness on bike sharing usage $[4,5]$. The main purpose of the research is to explore and compare the spatial associations between eye-level and overhead level greenness, and the usage of dockless bike sharing on weekdays, weekend, and holidays in a center area of Shenzhen, China [39-57].

Table 1. Studies related to cycling behavior and urban greenness.

\begin{tabular}{|c|c|c|c|c|}
\hline Topic & Author & Study Area & Methods & Research Questions or Viewpoint \\
\hline \multirow{7}{*}{$\begin{array}{l}\text { Spatial and temporal } \\
\text { analysis of dockless } \\
\text { bike sharing }\end{array}$} & Shen et al., 2018 [58] & Singapore & $\begin{array}{l}\text { Spatial autoregressive } \\
\text { model }\end{array}$ & $\begin{array}{l}\text { Link between bike use and built } \\
\text { environment }\end{array}$ \\
\hline & $\begin{array}{l}\text { Mooney et al., } 2018 \\
\text { [59] }\end{array}$ & Seattle & Descriptive statistics & $\begin{array}{l}\text { Comparison of freedom from fixed station } \\
\text { to dockless bike sharing }\end{array}$ \\
\hline & $\begin{array}{l}\text { Nickkar et al., } 2019 \\
{[60]}\end{array}$ & Baltimore & $\begin{array}{l}\text { Multinomial logistic } \\
\text { regression }\end{array}$ & $\begin{array}{l}\text { Link between bike use and gender and } \\
\text { land use }\end{array}$ \\
\hline & $\begin{array}{l}\text { Istiak A et al., } 2019 \\
\text { [61] }\end{array}$ & Baltimore & $\begin{array}{l}\text { Bike equity index and } \\
\text { traffic stress index }\end{array}$ & $\begin{array}{c}\text { Proposed indexes to prioritize bikeshare } \\
\text { infrastructure }\end{array}$ \\
\hline & Xu et al., 2019 [62] & Singapore & $\begin{array}{l}\text { Eigen decomposition } \\
\text { methods }\end{array}$ & $\begin{array}{l}\text { Proposed a framework to unravel the } \\
\text { landscape and pulses of bike sharing }\end{array}$ \\
\hline & Zhu et al., 2020 [63] & Singapore & $\begin{array}{l}\text { Methods to construct } \\
\text { the paths and estimated } \\
\text { repositioning trips and } \\
\text { the fleet sizes }\end{array}$ & $\begin{array}{l}\text { Spatial and temporal heterogeneity and } \\
\text { mobility of bike sharing }\end{array}$ \\
\hline & Gao et al., 2021 [2] & Shenzhen & GeoDetector model & $\begin{array}{l}\text { Modifiable areal unit problem in bike } \\
\text { sharing usage influential mechanism }\end{array}$ \\
\hline \multirow{11}{*}{$\begin{array}{l}\text { Urban greenness and } \\
\text { its relationship with } \\
\text { spatiotemporal } \\
\text { behaviors }\end{array}$} & $\begin{array}{c}\text { Lachowycz et al., } 2011 \\
\text { [27] }\end{array}$ & / & Systematic review & $\begin{array}{l}\text { Green space contributes to reducing } \\
\text { obesity and improving health. }\end{array}$ \\
\hline & Fraser et al., 2011 [13] & / & Systematic review & $\begin{array}{c}\text { Proximity of green space was positively } \\
\text { related to cycling }\end{array}$ \\
\hline & Krenn et al., 2014 [19] & Graz & GIS spatial analysis & $\begin{array}{l}\text { Bicyclists often used bicycle lanes and } \\
\text { pathways, flat, and green areas }\end{array}$ \\
\hline & $\begin{array}{l}\text { Cole-Hunter et al., } \\
\qquad 2015 \text { [28] }\end{array}$ & Barcelona & $\begin{array}{l}\text { Multinomial logistic } \\
\text { regression }\end{array}$ & $\begin{array}{l}\text { Greenness was positively related to } \\
\text { willingness of cycling commuting }\end{array}$ \\
\hline & $\begin{array}{l}\text { Christiansen et al., } \\
\qquad 2016 \text { [24] }\end{array}$ & 14 cities globally & $\begin{array}{l}\text { Generalized additive } \\
\text { mixed models }\end{array}$ & $\begin{array}{l}\text { Urban greenness was not associated } \\
\text { significantly with cycling frequency }\end{array}$ \\
\hline & Chen et al., 2017 [23] & Seattle & $\begin{array}{l}\text { Generalized linear } \\
\text { mixed model }\end{array}$ & $\begin{array}{c}\text { Urban greenness can attract cycling } \\
\text { activities }\end{array}$ \\
\hline & Sun et al., 2017 [32] & Glasgow & $\begin{array}{l}\text { Linear mixed-effects } \\
\text { model }\end{array}$ & $\begin{array}{l}\text { No association between cycling and } \\
\text { participants' proximity to green space }\end{array}$ \\
\hline & Marcin et al., 2018 [64] & $\begin{array}{c}\text { Lodz } \\
\text { (Poland) }\end{array}$ & Geoprocessing functions & $\begin{array}{l}\text { Large differences in the total amount of } \\
\text { green spaces in different datasets }\end{array}$ \\
\hline & Porter et al., 2019 [31] & $\begin{array}{l}4 \text { cities in United } \\
\text { States }\end{array}$ & $\begin{array}{l}\text { Logistic and negative } \\
\text { binomial regression }\end{array}$ & $\begin{array}{l}\text { Tree canopy coverage and parks positively } \\
\text { affect cycling frequency }\end{array}$ \\
\hline & $\begin{array}{l}\text { Łaszkiewicz et al., } \\
\qquad 2020 \text { [65] }\end{array}$ & $\begin{array}{l}\text { Lodz } \\
\text { (Poland) }\end{array}$ & Expert evaluation & $\begin{array}{c}\text { Mapping the social functions of urban } \\
\text { green spaces }\end{array}$ \\
\hline & $\begin{array}{l}\text { Khalaim et al., } 2021 \\
\text { [66] }\end{array}$ & Kyiv (Ukraine) & $\begin{array}{l}\text { A low-cost greenery } \\
\text { inventory method }\end{array}$ & $\begin{array}{c}\text { Urban trees contribute to the mitigation of } \\
\text { urban heat impacts }\end{array}$ \\
\hline \multirow{3}{*}{$\begin{array}{l}\text { Association between } \\
\text { greenness and cycling } \\
\text { or dockless bike } \\
\text { sharing }\end{array}$} & Lu et al., 2019 [3] & Hongkong & Logistic regression & $\begin{array}{l}\text { Eye-level greenness was positively related } \\
\text { to cycling }\end{array}$ \\
\hline & Wang et al., $2020[4]$ & Shenzhen & Poisson regression & $\begin{array}{c}\text { Eye-level greenness was positively related } \\
\text { to bike usage around metros }\end{array}$ \\
\hline & Chen et al., 2020 [5] & Shenzhen & $\begin{array}{l}\text { Multiple linear } \\
\text { regression }\end{array}$ & $\begin{array}{l}\text { Eye-level greenness was more related to } \\
\text { bike usage than NDVI }\end{array}$ \\
\hline
\end{tabular}




\section{Data and Methods \\ 3.1. Study Area}

The study area was located in the center of Shenzhen, China (Figure 1). It covers over $162 \mathrm{~km}^{2}$ with 101 metro stations, a high population density, and economy activities. The study area covers part of the Nanshan, Futian, and Luohu districts, the polycentricity of Shenzhen city, whose center areas (CBD, central business district) are also mapped in Figure 1. Nanshan CBD mainly covers Shenzhen University (the largest university) and high-tech parks with prominent high-tech enterprises such as HUAWEI, Tencent, DJI, and ZTE. Futian CBD has the largest number of well-known enterprise headquarters and the highest gross domestic product (GDP) in China. The main commercial sector in the Futian CBD is the financial industry, which is distributed with the Shenzhen Securities Exchange Center and many banks and financial enterprises. Luohu CBD also serves as a financial center in Shenzhen. Both the Futian CBD and Luohu CBD are near checkpoints connecting Hong Kong, China. All have a high population density and numerous economic activities. Shenzhen is one of the most developed and innovative cities in China, and it also enjoys early, stable, and continuous operation of dockless bike sharing. Therefore, Shenzhen's bike sharing system has become a research focus among bike sharing researchers [2,4,5,39].

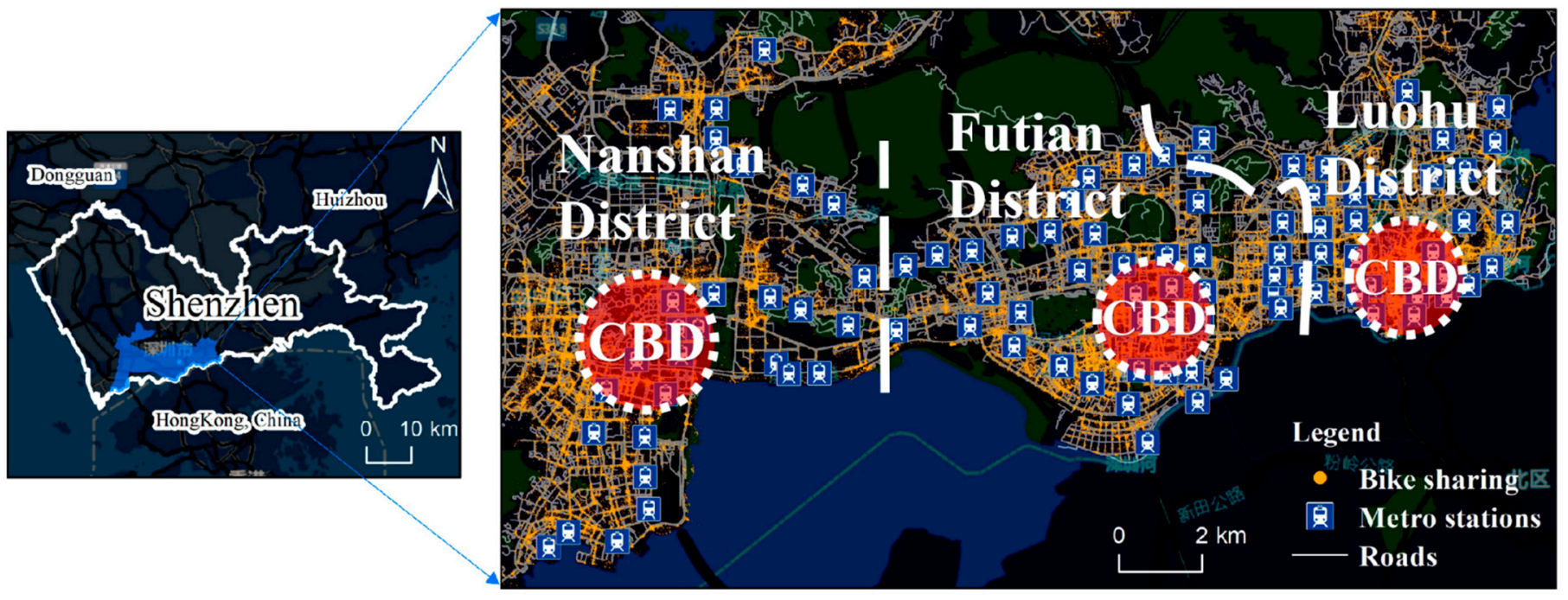

Figure 1. Study area. CBD, central business district.

To explore the association between urban greenness and dockless bike sharing usage for metro-bike transfer travel, $1 \mathrm{~km}$ radii buffers around metro stations (Figure $2 \mathrm{a}$ ) were applied in this study, based on the framework for inferring the travel purpose of bike sharing users in the gravity model and Bayesian rules proposed by Li et al. (2021) [39]. Then, the overlapping problem is avoided using the Thiessen-polygon method [4]. Finally, the Thiessen-polygon buffers were clipped by the coastline and the boundary with Hong Kong, China (Figure 2b).

\subsection{Data}

The data used in the study can be categorized into three types: dockless bike sharing, urban greenness, and other built environment factors.

Dockless bike sharing record data were obtained from the bike sharing operators in 2018, including most major brands such as Mobike, Ofo, Bluegogo, and Xiaoming Bike. The bike data record every trip's location and time information of both origin and destination, ranging from 1 October to 14 October 2018 (including a 7 day public holiday, 5 day week, and a 2 day weekend). The raw dataset was pre-processed by clearing redundant records and excluding unrealistically long or short distances or durations [2]. Given that this study focused on the date variation in the association of urban greenness and bike sharing usage, 
the bike dataset was grouped by weekdays, weekends, and holidays. The hourly usage of weekdays, weekends, and holidays was quantified by the integration of both origin and destination amounts.
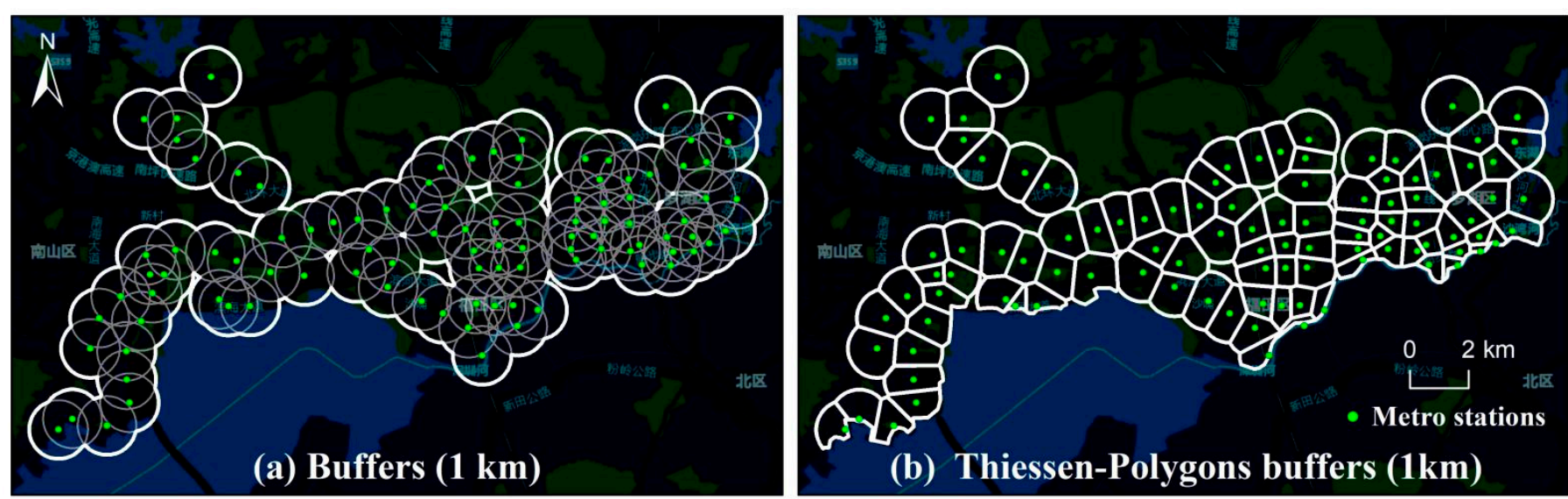

Figure 2. (a) Buffers and (b) Thiessen-polygon buffers.

Urban greenness indicators were separated into two types: eye-level urban greenness assessed by street view images and deep learning segmentation, and NDVI from Landset8 images. A total of 40,272 street view images from 10,068 sampling points (every sampling point collected from street view images from four directions for a $360^{\circ}$ view image) were obtained via BMap API (http:/ /lbsyun.baidu.com/, accessed on 1 October 2018) in 2018. The street-view images were further segmented by a fully convolutional neural network for semantic image segmentation $[4,35,37,40]$ and the ADE20 K training dataset of labeled images. Figure 3 shows examples of eye-level urban greenness assessed using the procedure mentioned above.

Street-view greenness was measured based on the segmentation results of street-view images. The ratio of greenness pixels to total pixels of street view images from four angles $\left(360^{\circ}\right)$ of each sampling point (Figure 3 ) was applied to measure the urban greenness at eye-level, which can be expressed as follows [3].

$$
G V I=\frac{\sum_{i=1}^{4} \text { Greenness Pixels }}{i}
$$

Greenness view index (GVI) ranged from 0 to 1 with higher values indicating higher levels of eye-level urban greenness [3].

Other built environment factors include densities of road intersections, primary roads, secondary roads, branch roads, bus stops, and buildings, as well as floor area ratio, land use entropy, retail density, scenic spot density, and real-time population density. Road intersections' density, primary road density, secondary road density, and branch road density were obtained from OpenStreetMap (https://www.openstreetmap.org/, accessed on 1 October 2018). Building density and floor area ratio were assessed based on the building footprint data obtained from BMap (http://lbsyun.baidu.com/, accessed on 1 October 2018). Bus stops, retail density, and scenic spot density were extracted from the points of interests (POIs) dataset from the BMap API (http://lbsyun.baidu.com, accessed on 1 October 2018). In addition, land use entropy was calculated based on the POIs mentioned above. Finally, the real-time population density, specifically, the Tencent user's density, was obtained from the Tencent LBS (location-based service) service platform (https:/ / heat.qq.com/document.php, accessed on 1 October 2018) accessed on 1 in October 2018, which has been widely used in various fields of study [2,41-43]. The raw hourly real-time population density data were then grouped and calculated as hourly values for weekdays, weekends, and holidays. 


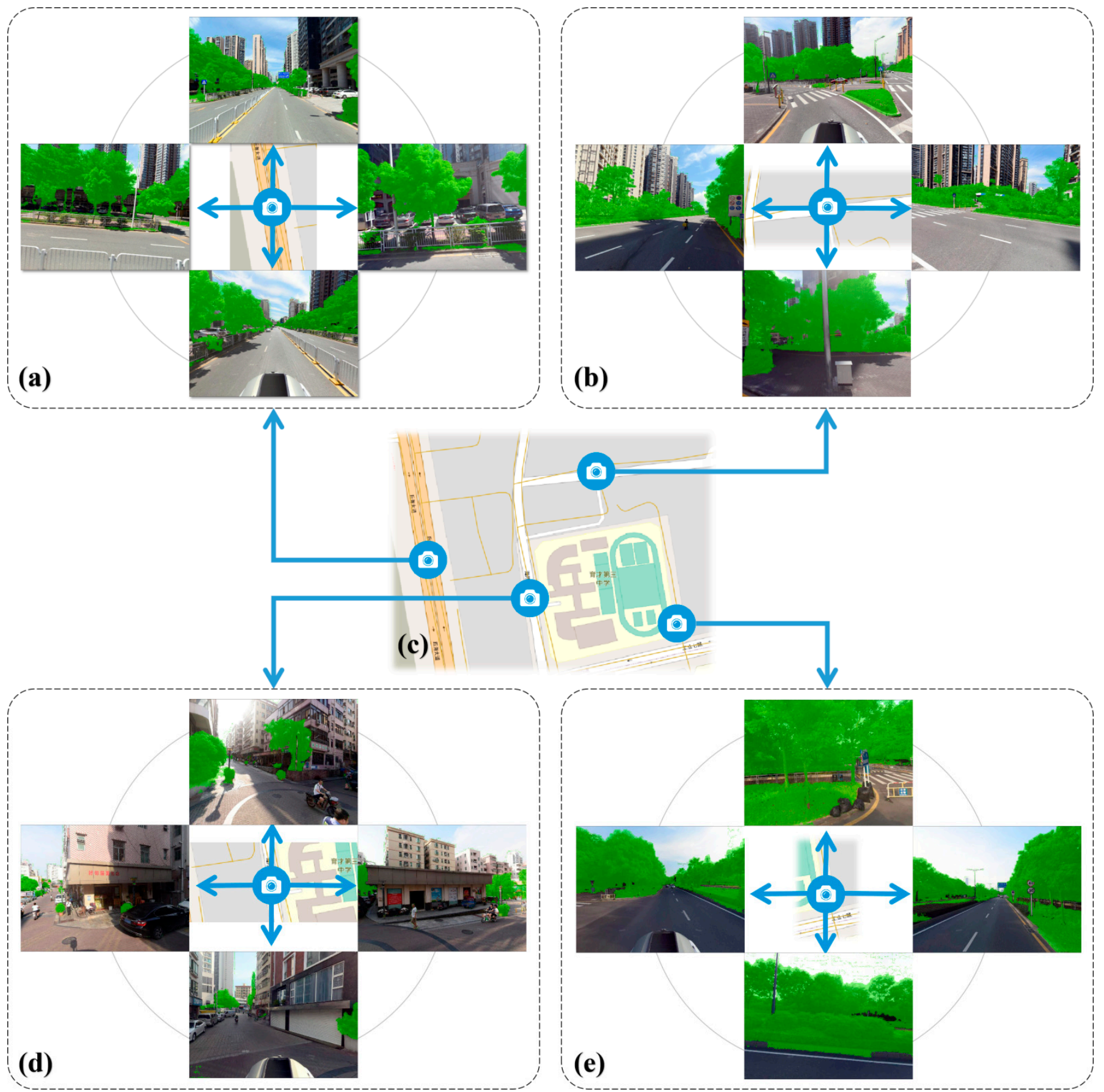

Figure 3. (a) The eye-level greenness at point a. (b) The eye-level greenness at point b. (c) The spatial distribution of four sampling points. (d) The eye-level greenness at point d. (e) The eye-level greenness at point e.

\subsection{Variable Selection and Geographically Weighted Regression (GWR)}

A related study proved that the combination of stepwise linear regression and GWR can effectively explore the spatially varying effects of independent variables on a dependent variable, especially in transport geography analysis, for the following two reasons [44]. Firstly, backward stepwise linear regression can effectively solve the problem of multicollinearity [44]. Second, after filtering independent variables, GWR can help explore the spatial heterogeneity of the association between urban greenness and dockless bike sharing usage. 
The GWR model is a type of regression model that considers spatial autocorrelation [44-47]. Because it integrates the spatial variation into coefficient estimation, it has the advantage of better performance in regression results [44-47]. The GWR model can be expressed as follows:

$$
y_{i}=\beta_{0}\left(u_{i}, v_{i}\right)+\sum \beta_{k}\left(u_{i}, v_{i}\right) x_{i k}+\varepsilon_{i}
$$

where $i$ represents the Thiessen-polygon buffer, $y_{i}$ represents the dependent variable (dockless bike sharing usage), $\left(u_{i}, v_{i}\right)$ represents the spatial location of $i, 0$ represents the intercept, $k$ represents the coefficients, $x_{i k}$ represents the independent variables, and $i$ represents the error term [44-47]. The GWR model estimator can be expressed as follows:

$$
\beta^{\prime}=\left[X^{T} W\left(u_{i}, v_{i}\right) X\right]^{-1} X^{T} W\left(u_{i}, v_{i}\right) Y
$$

where $W\left(u_{i}, v_{i}\right)$ represents the square matrix of weights, which is related to the spatial location of $\left(u_{i}, v_{i}\right) ; X^{T} W\left(u_{i}, v_{i}\right) X$ represents the geographically weighted variance; and $\mathrm{Y}$ is the vector of values for the dependent variable [44-47].

The $W\left(u_{i}, v_{i}\right)$ matrix consists of geographical weights in its leading diagonal and zero in its off-diagonal elements [44-47]:

$$
W_{\left(u_{i}, v_{i}\right)}=\left[\begin{array}{ccc}
W_{1}\left(u_{i}, v_{i}\right) & 0 & 0 \\
0 & \ldots & 0 \\
0 & 0 & W_{n}\left(u_{i}, v_{i}\right)
\end{array}\right]
$$

where $n$ represents the number of Thiessen-polygon buffers in this study.

As Tobler's first law of geography states that "everything is related to everything else, but near things are more related than distant things" [48], it is assumed that the influence of near observations on parameter estimation is greater than that of distant observations, which indicates that the influence decreases with an increase in distance [49]. The determination of the kernel bandwidth is crucial for GWR because it greatly affects the model results [49]. Given that the spatial distribution of dockless bike sharing usage varied across the study area, the adaptive spatial kernel was applied in the study for geographical weighting [50]. Additionally, according to the previous study, the corrected Akaike information criterion (AICc) method was adopted to determine the bandwidth [51]. In conclusion, GWR has remarkable advantages over global regression in that it is capable of exploring the spatially varying impacts of potential driving factors on the dependent variable, including both coefficients and diagnostics, which helps improve the relationship studies in model performance and comprehensive interpretation.

Figure 4 presents the study workflow. Bike sharing usage, landsat 8 images, and street view images were major data sources, and the integration of backward stepwise regression and GWR was applied to explore the spatial association between dockless bike-sharing usage and urban greenness. Finally, urban planning implications for cycling-friendly environments were promoted based on the GWR results. 


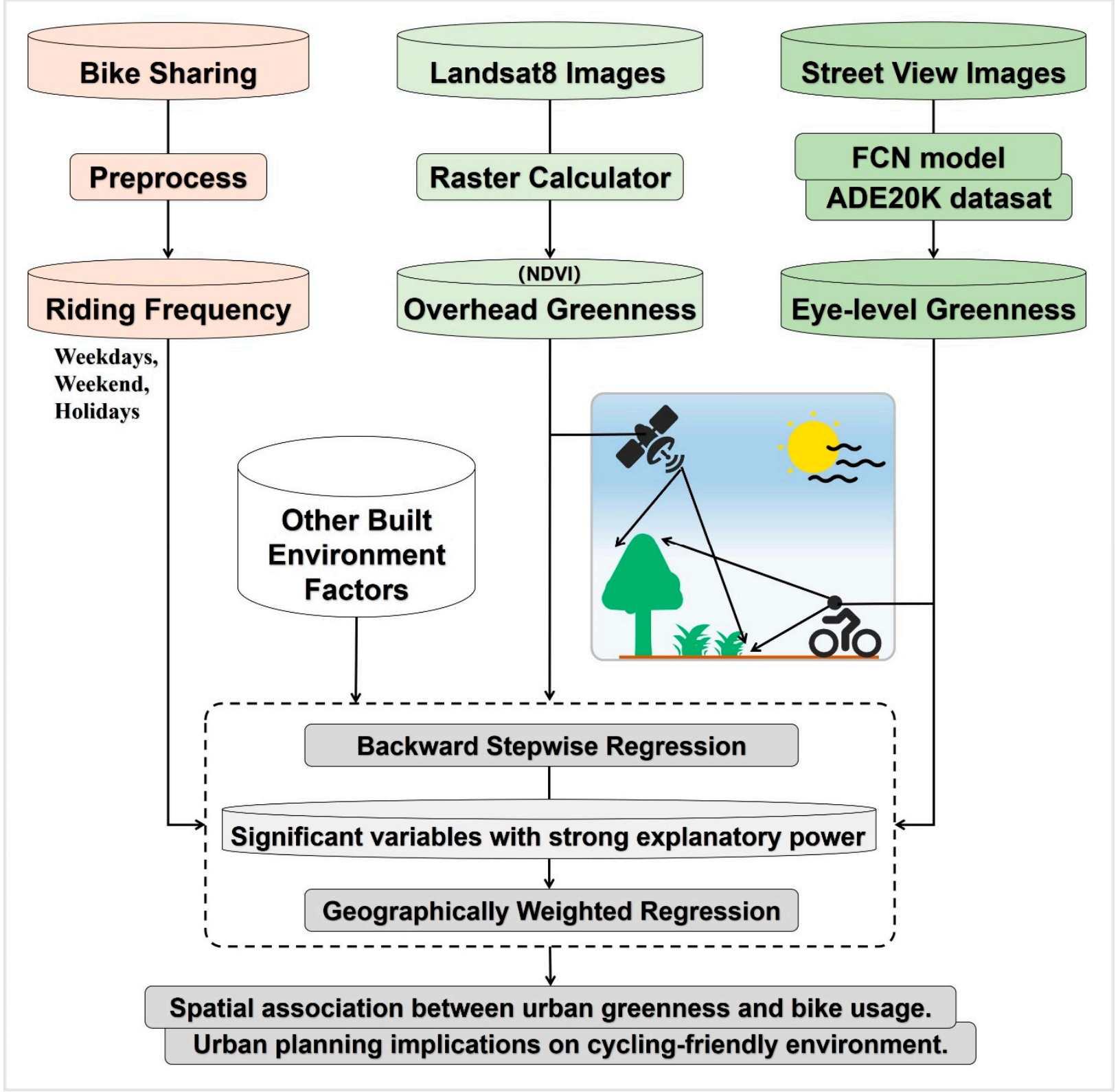

Figure 4. Study workflow.

4. Results and Discussion

4.1. Dockless Bike Sharing Usage and Urban Greenness

This study aims to explore the association between dockless bike sharing usage on weekdays, weekends, and holidays (Figure 5) and urban greenness (Figure 6). Figure 5 presents the spatial distribution of dockless bike sharing usage on weekdays, weekends, and holidays. The hourly usage of dockless bike sharing on weekdays (6783) was the highest, followed by weekends (5851) and holidays (5115) (Figure 5d). Buffers with high cycling frequency were observed in the western, middle, and eastern parts of the study area, corresponding to the polycentricity of Shenzhen, including Nanshan, Futian, and Luohu districts [52]. Similarly, the spatial distributions of the bike sharing usage on weekdays, weekends, and holidays share the same polycentricity patterns, despite the variation in bike usage volume [2]. 

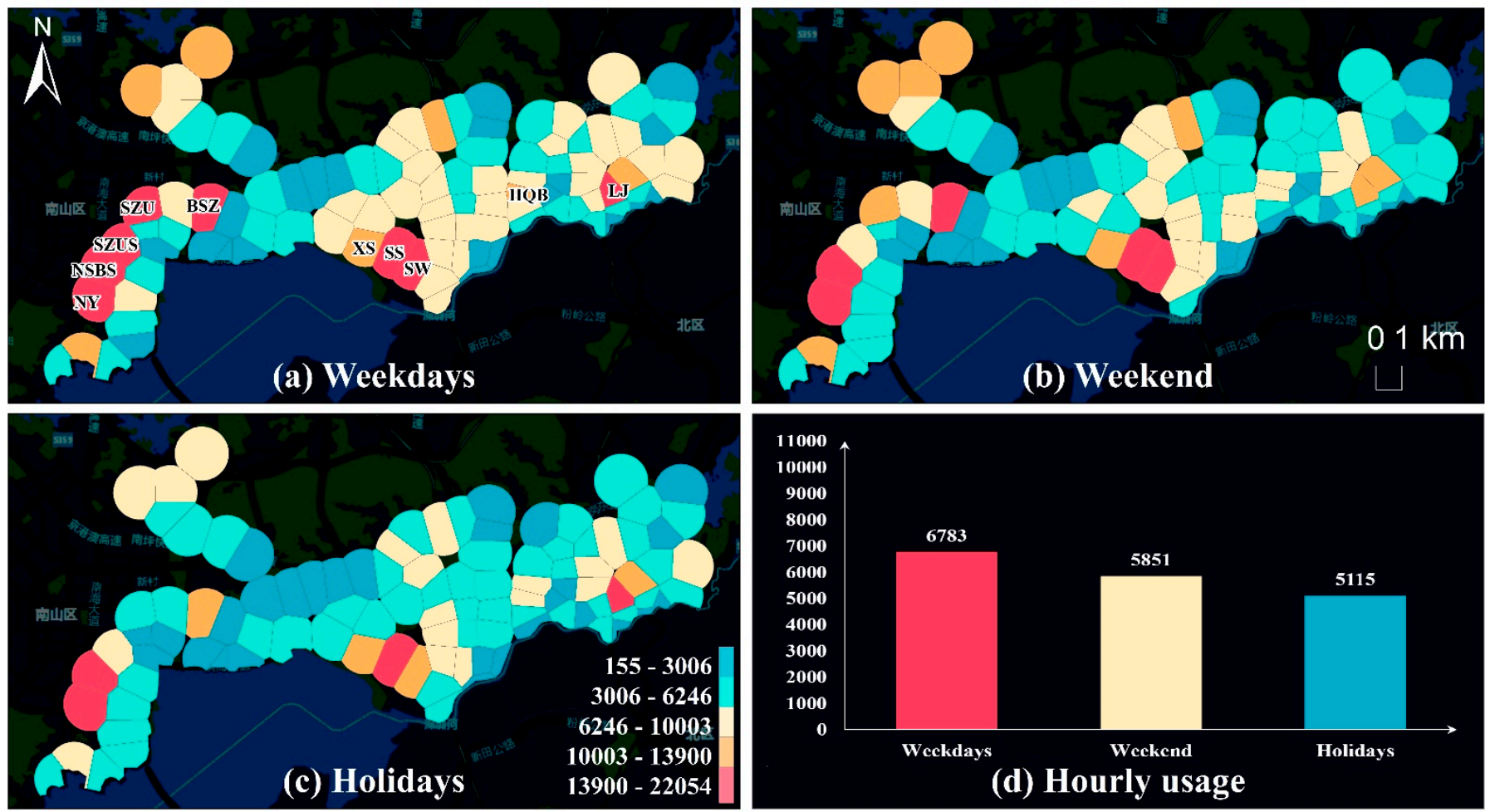

Figure 5. Dockless bike sharing usage on (a) weekdays, (b) weekend, and (c) holidays. (d) The hourly usage on weekdays, weekend and holidays.
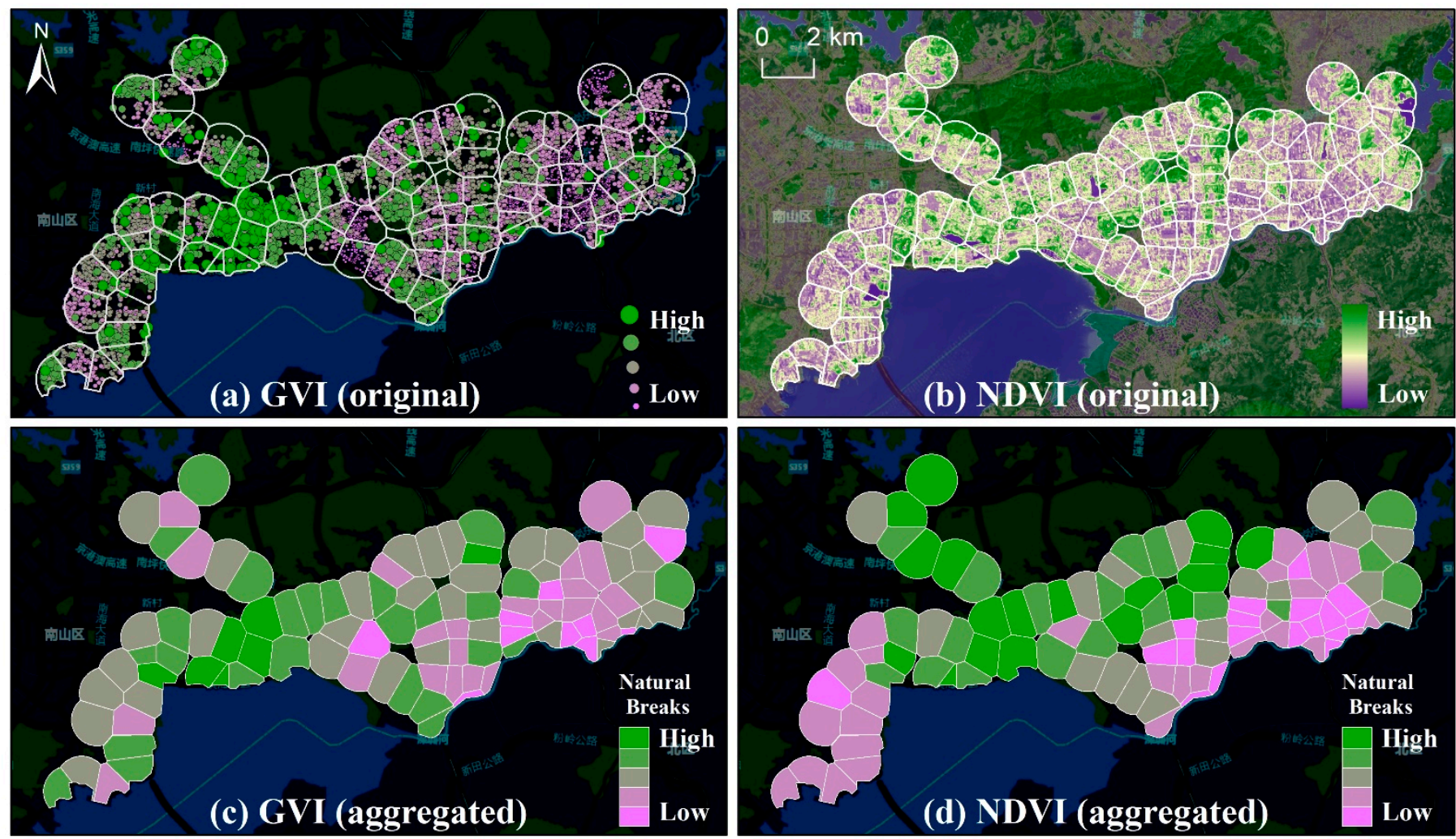

Figure 6. (a) Spatial distribution of eye-level greenness at each sampling points. (b) Spatial distribution of overhead view urban greenness (NDVI). (c) Spatial distribution of aggregated eye-level greenness. (d) Spatial distribution of aggregated NDVI. 
On weekdays, high cycling frequency in Nanshan district mainly occurred in the CBD, including SZU (Shenzhen University), SZUS (Shenzhen University South), NSBS (Nanshan Book Shop), NY (Nanyou industrial zone), and BSZ (Bai Shizhou). Among them, SZU and SZUS are near the largest university and high-tech parks, while the NY metro station mainly serves the local petroleum industrial zone. In Futian district, high usage occurred in the southwestern part of its CBD (SS and SW metro station), rather than inside the CBD. It is an area with a distribution of high-density urban villages. For the Luohu district, its high usage was in the CBD core, including the LJ metro station and the old town of Shenzhen, where the first $\mathrm{CBD}$ was developed without specific planning suggestions. Moreover, high bike sharing usage was also observed around the HQB station on weekdays, which is the mobile phone retail and wholesale industry center. It was noted that high usage around the HQB station only existed on weekdays. The high usage of bike sharing on weekends and holidays was roughly consistent with that on weekdays. On weekends, the bike usage around SZU, SZUS in Nanshan district, and $\mathrm{Lj}$ in Luohu district was lower than usage on weekdays. On holidays, the number of metro stations with high bike usage was the lowest, with only four stations.

Figure 6 shows the spatial distribution of two urban greenness indicators in the study, eye-level greenness extracted from street view images and overhead view greenness from NDVI. Both the original data (Figure $6 a, b$ ) and aggregated data based on Thiessen-polygon buffers (Figure 6c,d) are presented below.

From the comparisons in Figure 6, it can be noted that the spatial distributions of eye-level greenness and overhead view greenness are partly consistent. The overhead view greenness distribution tended to be spatially continuous, whereas eye-level greenness was relatively discrete. The high greenness from the overhead view was mainly located in the middle and northern regions, and that from eye-level was mainly distributed in the central and western regions and scattered in other regions. Notably, there were some similarities, but also obvious differences between the two urban greenness indicators in measuring urban greenness distribution with mutually perpendicular perspectives. Therefore, two urban greenness indicators are applied in the next sections to further explore their association with dockless bike sharing usage on different dates.

\subsection{Stepwise Linear Regression and Variable Selection}

Table 2 presents the summary statistics for all variables, and the independent variables can be classified into four categories: urban greenness, traffic accessibility, built environment, and population distribution.

Multi-collinearity commonly exists in various linear regression models, which may generate misleading and insignificant results. Hence, backward stepwise linear regression was first applied in this study to solve the problem of multi-collinearity and select significant variables with strong explanatory power for the three models $[44,53,54]$. In addition, sixteen candidate variables were standardized with zero mean standardization to ensure that the mean was 0 and the standard deviation was $1[53,54]$. Table 3 shows the results of three backward stepwise linear regression models corresponding to weekdays, weekends, and holidays. It is clear that these results are significantly different. Street-view greenness (SVG), Sec.Road, and building density were included in all models. The results for weekends and holidays were similar to those of SVG, NDVI, Sec.Road, building density, floor area ratio (FAR), land use entropy (MIX), and real-time population density (POP) retained in the models. Moreover, scenic spots density retains only in the holiday model. Road.Int, Pri.Road, Bran.Road, Bus stops density (BUS), and retail stores density (RETAIL) were all excluded from the three models. 
Table 2. Summary statistics for all variables. NDVI, normalized difference vegetation index.

\begin{tabular}{|c|c|c|c|c|}
\hline Categories & Variables & Abbreviation & Mean & Std. Deviation \\
\hline \multirow{3}{*}{ Dependent variable } & Bike use on weekdays & / & 6783.491 & 4463.041 \\
\hline & Bike use on weekend & / & 5851.406 & 4156.571 \\
\hline & Bike use on holidays & / & 5115.133 & 3427.846 \\
\hline \multirow{2}{*}{ Urban greenness } & Greenness view index & GVI & 0.297 & 0.105 \\
\hline & NDVI & NDVI & 0.173 & 0.051 \\
\hline \multirow{5}{*}{ Traffic accessibility } & Road intersection density (number $/ \mathrm{km}^{2}$ ) & Road.Int & 1083.289 & 463.527 \\
\hline & Primary road density $\left(\mathrm{km} / \mathrm{km}^{2}\right)$ & Pri.Road & 3.265 & 2.657 \\
\hline & Secondary road density $\left(\mathrm{km} / \mathrm{km}^{2}\right)$ & Sec.Road & 9.180 & 4.000 \\
\hline & Branch Road density $\left(\mathrm{km} / \mathrm{km}^{2}\right)$ & Bran.Road & 8.185 & 4.169 \\
\hline & Bus stops density (number $/ \mathrm{km}^{2}$ ) & BUS & 45.302 & 30.066 \\
\hline \multirow{5}{*}{ Built environment } & Building density (\%) & Building & 0.197 & 0.080 \\
\hline & Floor area ratio & FAR & 1.706 & 0.897 \\
\hline & Land use entropy & MIX & 0.803 & 0.146 \\
\hline & Retailing POIs density (number $/ \mathrm{km}^{2}$ ) & RETAIL & 7.286 & 9.575 \\
\hline & Scenic spot POIs density (number $/ \mathrm{km}^{2}$ ) & SPOT & 5.373 & 6.428 \\
\hline \multirow{3}{*}{ Population distribution } & $\begin{array}{c}\text { Tencent user density } \\
\text { (weekdays, person } / \mathrm{km}^{2} \text { ) }\end{array}$ & POP & $90,395.701$ & $61,258.328$ \\
\hline & $\begin{array}{c}\text { Tencent user density } \\
\text { (weekend, person } / \mathrm{km}^{2} \text { ) }\end{array}$ & POP & $68,518.758$ & $39,405.458$ \\
\hline & $\begin{array}{l}\text { Tencent user density } \\
\text { (holidays, person } / \mathrm{km}^{2} \text { ) }\end{array}$ & POP & $65,986.565$ & $37,886.493$ \\
\hline
\end{tabular}

Table 3. The significant variables included in stepwise linear regression models. SVG, streetview greenness.

\begin{tabular}{cccc}
\hline Variables & Weekdays & Weekend & Holidays \\
\hline SVG & $-* * *$ & $-* * *$ & $-* * *$ \\
NDVI & & $-* *$ & $-* * *$ \\
Road.Int & & & \\
Pri.Road & $-* * *$ & $-* * *$ & $-* *$ \\
Sec.Road & & & $-* *$ \\
Bran.Road & $-* * *$ & $-* *$ \\
BUS & & $-* * *$ & $-* * *$ \\
Building & & $-* *$ & $-*$ \\
FAR & & $-* *$ & $-*$ \\
MIX & & $-*$ & \\
RETAIL & &
\end{tabular}

The results of backward stepwise linear regressions indicated that eye-level urban greenness, secondary road density, and building density were significantly important for the dockless bike sharing usage around metro stations on weekdays. In addition to the three variables mentioned above, NDVI, floor area ratio, land use entropy, and real-time population density were also significantly associated with bike sharing usage on weekends. The results of both weekdays and weekends are consistent with those of previous studies; however, the association between bike sharing usage and urban greenness on holidays has never been discussed and explored. It is common for residents to be more available and flexible on holidays to ride bike sharing for recreation and commuting purposes than on weekend and weekdays, and that work overtime usually exists on weekend rather than on public holidays (National Day Holidays of China) in China. Therefore, exploring the relationship between urban greenness and dockless bike sharing usage of holidays is of great importance for better understanding the usage mechanism of bike sharing and 
policymaking in a cycling-friendly environment. Here, we discovered some findings that have seldom been observed in related cases. For example, the significant weekend variables are consistent with those of holidays, except scenic spot density. Second, both eye-level greenness (SVG) and overhead view greenness (NDVI) were significantly associated with bike usage $(p \leq 0.01)$.

\subsection{Spatial Variation of Coefficients from GWR Models}

Previous studies on the association between urban greenness and cycling with global regression can quantify the impacts of independent variables, however, the estimated parameters are global and identical for all observations [3-5]. GWR is superior to global models as it can explore the spatial heterogeneity in explanatory variables' impacts $[44,47,55,56]$. The main purpose of this study was to assess the local influence coefficients of dockless bike sharing usage to compare the associations with bike usage between eye-level greenness and overhead view greenness with GWR. To further uncover the differences between weekdays, weekends, and holidays, three models were built. First, global spatial autocorrelation tests for three dependent variables were performed and measured using Moran's I $[44,47]$. Table 4 shows that spatial autocorrelation was found for dockless bike sharing usage on weekdays, weekends, and holidays with $p$-values lower than 0.05 , indicating that the dependent variables were spatially clustered.

Table 4. Moran's I of the dependent variables.

\begin{tabular}{cccccc}
\hline Variables & Moran's I & Expected I & Pattern & z-Score & $\boldsymbol{p}$-Value \\
\hline Bike use on weekdays & 0.261366 & -0.009804 & Clustered & 4.574797 & 0.000005 \\
Bike use on weekend & 0.225562 & -0.009804 & Clustered & 3.961404 & 0.000075 \\
Bike use on holidays & 0.228662 & -0.009804 & Clustered & 4.015384 & 0.000059 \\
\hline
\end{tabular}

Table 5 presents the estimated parameters of backward stepwise linear regression and GWR models for weekdays, weekends, and holidays. Initially, the Moran's I for residuals of all three backward stepwise linear regression models was significantly positive (Moran's I > 0.2, $p$-value $=0.001$ ), which suggests that the global regression assumption of residual independence was invalid. Therefore, GWR can be applied to solve this problem [44,47,55-57]. The results were significantly improved by GWR in the following aspects (Table 5). Firstly, the adjusted R-squared values improved from 0.433 to 0.636 in the weekdays model, from 0.458 to 0.527 in the weekend model, and from 0.507 to 0.522 in the holidays model. This indicates that the GWR model explains $63.6 \%$ of dockless bike sharing usage on weekdays, $52.7 \%$ on weekends, and $52.2 \%$ on holidays. Specifically, GWR enhanced the adjusted R-square of the three models by $21.6 \%$ on average. This is a remarkable improvement when neighboring observations are considered in the regression process, which is the most important feature of GWR. Secondly, the AICc of the weekdays model declined from 220.333 in the global model to 204.794 in the GWR, and that of the weekend and holidays models also decreased.

Table 5. Stepwise linear regression (global) and geographically weighted regression (GWR) diagnostics.

\begin{tabular}{ccccccc}
\hline \multirow{2}{*}{ Diagnostics } & \multicolumn{2}{c}{ Weekdays } & \multicolumn{2}{c}{ Weekend } & \multicolumn{2}{c}{ Holidays } \\
\cline { 2 - 7 } & Global & GWR & Global & GWR & Global & GWR \\
\hline R-Squared & 0.450 & 0.753 & 0.495 & 0.615 & 0.546 & 0.594 \\
Adjusted R-Squared & 0.433 & 0.636 & 0.458 & 0.527 & 0.507 & 0.522 \\
AICc & 220.333 & 204.794 & 217.77 & 212.503 & 200.335 & 196.533 \\
Moran's I & 0.262 & 0.039 & 0.276 & 0.092 & 0.221 & 0.007 \\
(residuals) & $(0.00)^{*}$ & $(0.41)$ & $(0.00) *$ & $(0.26)$ & $(0.00)^{*}$ & $(0.32)$ \\
\hline
\end{tabular}

Note: $p$-value are given in brackets; ${ }^{*}$ statistically significant at level: $\alpha=0.05$. AICc, corrected Akaike information criterion. 
The GWR model is also capable of identifying the areas that best fit the model based on the local R-square [57]. Hence, the model's local performance to explore the dockless bike sharing usage of different metro station buffers can be visualized. Figure 7 presents the local R-square of the GWR models of weekdays, weekends, and holidays. The spatial distributions of the local R-square of three models are non-homogeneous, which indicates that the GWR model performance differs in different areas. For instance, the GWR model performance of weekdays was satisfactory in the middle near the bay in the study area, with local R-square higher than 0.667. Meanwhile, the northwestern and northeastern regions had a lower local R-square, suggesting that there might be extra explanatory variables affecting the bike sharing usage in those areas.
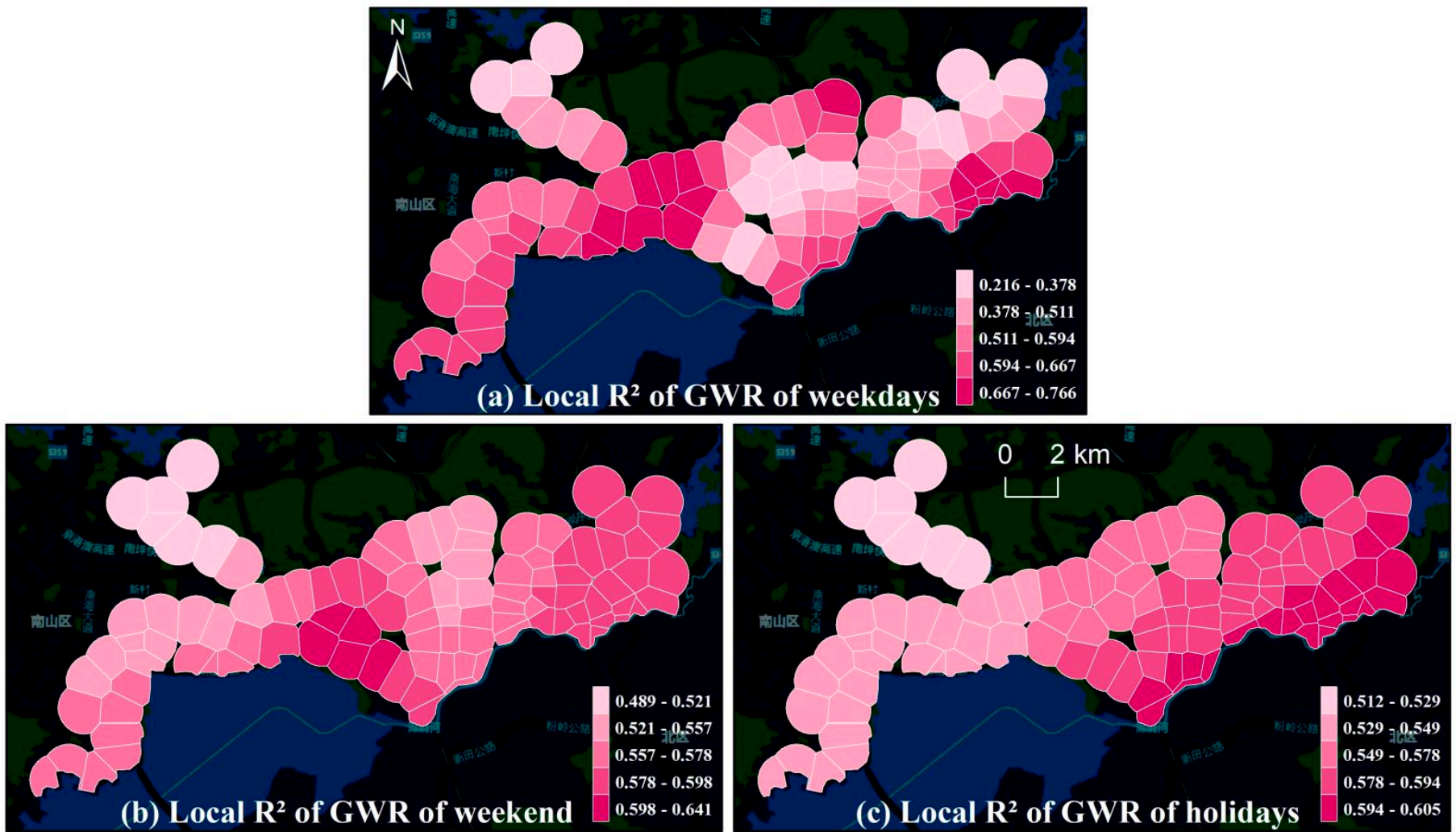

Figure 7. Spatial distribution of local $\mathrm{R}^{2}$ for the geographically weighted regression (GWR) models of weekdays (a), weekend (b) and holidays (c).

\subsubsection{Urban Greenness and Bike Sharing Usage around Metro Stations}

Previous studies explored the association between greenness and bike sharing usage or cycling activity frequency using global models, such as logistic regression [3], Poisson regression model [4], and multiple linear regression [5]. All were incapable of further exploring the spatial heterogeneity of the local association between greenness and cycling [3-5]. This study attempts to solve this research gap by applying the GWR model, with which the spatially varying effects of urban greenness indicators as well as other built environment factors on dockless bike sharing usage are presented as follows.

Figure 8 shows the local coefficients of urban greenness indicators (eye-level greenness and overhead view green-ness) in GWR models for weekdays, weekends, and holidays, which can reflect the local association between bike sharing usage and urban greenness indicators. On the one hand, Figure 7a,b show that overhead view greenness (NDVI) was negatively associated with the bike sharing usage of all metro station buffers on weekends and holidays, while it was insignificant during weekdays. The negative effect mainly existed in Nanshan district and the northern part of Futian district on weekends, while it was mainly distributed in Nanshan district on holidays. 

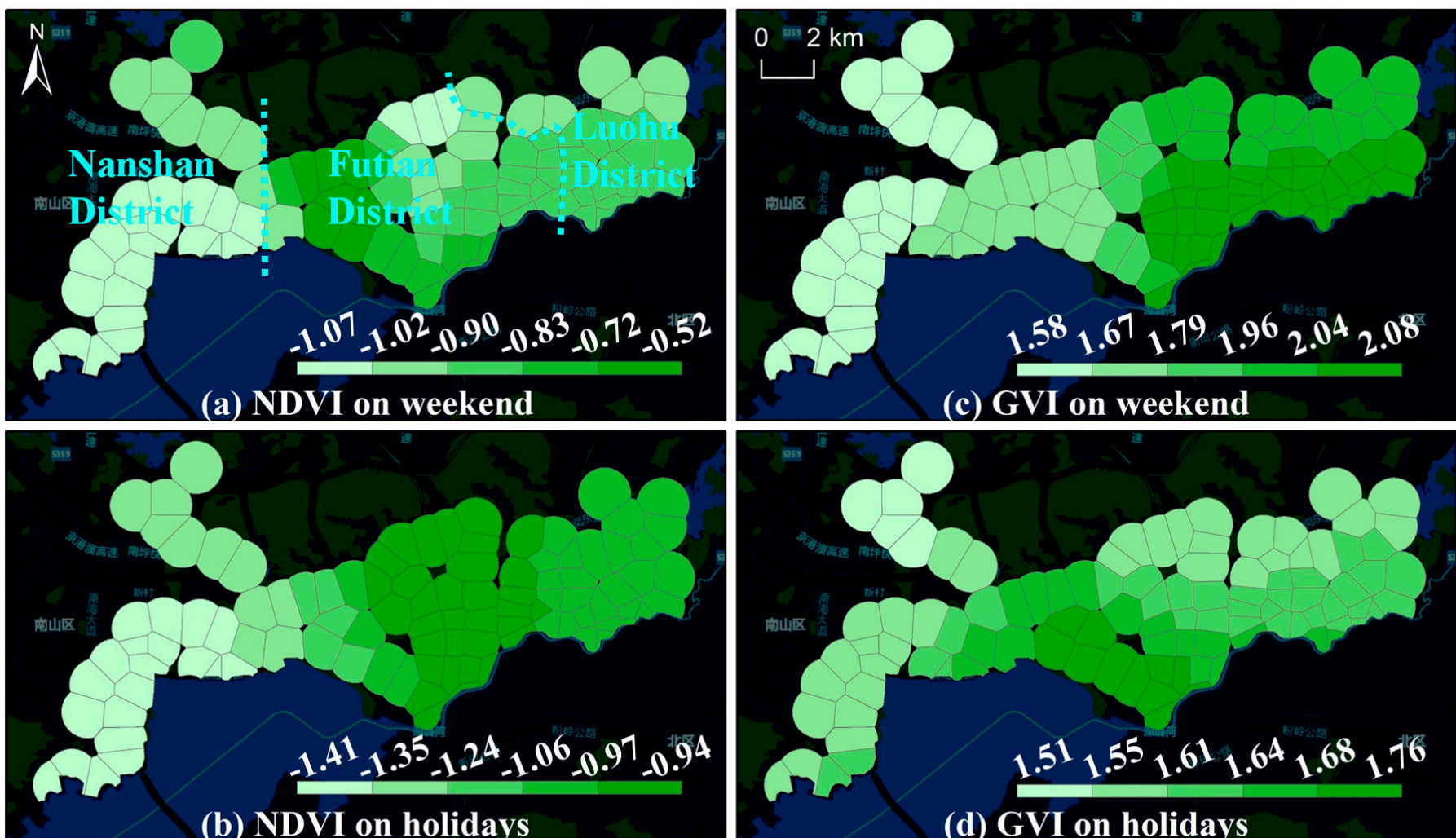

(b) NDVI on holidays

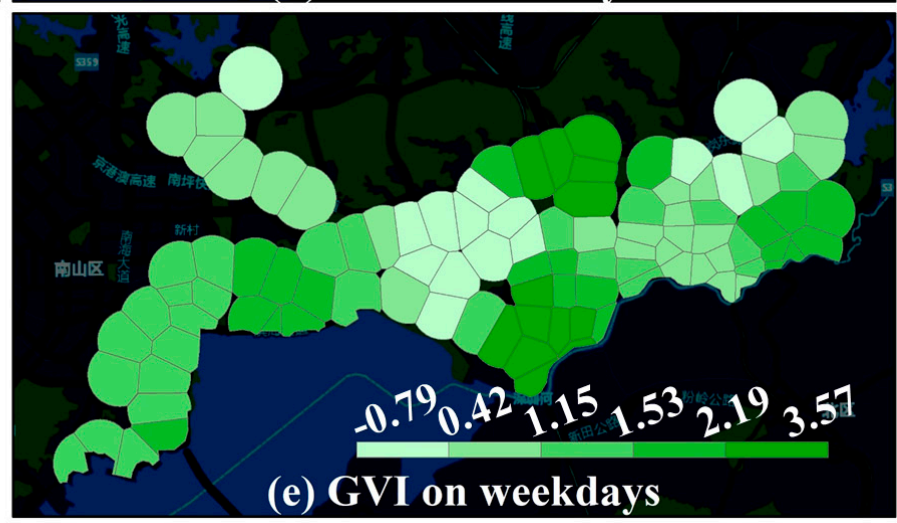

Figure 8. Local coefficients of NDVI on weekend (a) and holidays (b). Local coefficients of eye-level greenness on weekend (c), holidays (d) and weekdays (e).

However, in contrast, eye-level greenness was positively related to bike sharing usage in most cases, except several observations on weekdays. The spatial distribution of this positive association of weekends, holidays, and weekdays was different from that of weekends, and holidays tended to be more clustered than the weekdays. On weekends, a strong positive association was observed in Luohu district and western Futian district, and a decreasing trend from east to west was also observed. On holidays, the positive association was focused in the eastern and southern regions of Futian district. Generally, the northern and western study area regions had relatively weak associations. On weekdays, the positive association distribution tended to be polycentric; that is, it centered on the northern Futian district, southern Futian district, southwestern Luohu district, and eastern Nanshan district. Therefore, attention should be paid to eye-level street view greenness in urban planning and design, rather than overhead view greenness, to build a cycling-friendly environment and promote the usage of dockless bike sharing and public transportation. Moreover, eye-level greenness in the southern and eastern parts of the study area should be well 
designed and developed, where strong positive association between it and bike sharing usage exists on weekdays, weekends, and holidays.

In conclusion, two urban greenness indicators were adopted, eye-level greenness and overhead view greenness, to explore and compare their association with dockless bike sharing usage on weekdays, weekend, and holidays. Overhead view greenness (NDVI) was found to have a significantly negative association with bike usage on weekends and holidays, and it was insignificant in the model of weekdays.

The difference in the impacts of these two urban greenness indicators can be attributed to the following reasons. In general, overhead view urban greenness contributes less to the encouragement of bike sharing usage, which might be because the NDVI obtained from the overhead perspective might differ from the view of cyclists. In addition, some types of urban greenness are ignored from an overhead perspective, such as vertical green walls and green spaces under overpasses [3], while some misleading greenness from NDVI can be excluded from eye-level greenness such as green roofs. Moreover, the positive association between eye-level greenness and bike sharing usage indicates that urban greenness obtained at human eye-level is closely related to cycling, as it is more in line with people's real feelings and experiences about urban greenness, which is consistent with previous studies [3-5]. Overall, this study is the first time among related studies that spatially varying association between eye-level greenness and bike sharing usage was explored on holidays in addition to weekdays and weekend with the GWR model.

\subsubsection{Other Built Environment Factors}

Figure 9 shows the local coefficients of secondary road density and building density in the GWR models for weekdays, weekends, and holidays. In addition to GVI, secondary road density and building density are significantly positively associated with dockless bike sharing usage for all time periods considered.

Secondary road density was the only significant variable in the traffic accessibility category among primary road, branch road, and intersections as well as bus stops densities. This suggests that most dockless bike sharing was used along secondary roads, rather than primary and branch roads. Figure $9 \mathrm{a}-\mathrm{c}$ show the local coefficients, from which it can be noted that the strong associations mainly exist in Nanshan district continuously. Specifically, on weekdays, strong associations can be observed in the Nanshan district, as well as western and eastern Futian districts. Relatively weaker associations were found in the middle Futian and southern Luohu districts. On weekends, strong associations were found in similar locations as on weekdays. On holidays, the distribution of local Sed.Road coefficients decreased from west to east in the study area. Notably, the junction of Nanshan and Futian districts was the area with consistently high local coefficients, which might reflect the high integration between Nanshan and Futian districts, and residents of the two regions might often travel across the regions by dockless bike sharing along the secondary roads closely connecting both sides. It was shown that secondary roads are essential infrastructure for cycling; hence, to promote the usage of bike sharing, cycling facilities along secondary roads should be well planned and built, such as cycle paths, bike parking areas, and path levelling. In addition, as mentioned above, greenness should also be designed and planted along the secondary roads from an eye-level perspective. Related studies only considered road intersections as a variable in the form of unclassified points without classifications [3-5], which are too dispersed and difficult to use when make spatially accurate planning suggestions.

The local coefficients of building density in the three models are presented in Figure $9 d-f$, which are significantly positively associated with bike sharing usage all the time. It is remarkable that the distribution of strong associations between building density and bike usage moved from the eastern part of Nanshan district, to the junction between the two districts, and to Futian district, corresponding to weekdays, weekends, and holidays. This was partly consistent with the secondary road density distribution of local coefficients, which indicates that attention should focus on secondary road density and building density. 
Additionally, the high level of the building density coefficients also reflects that areas with high buildings density usually tend to be comprehensively well developed and demands for bike usage might be generated.

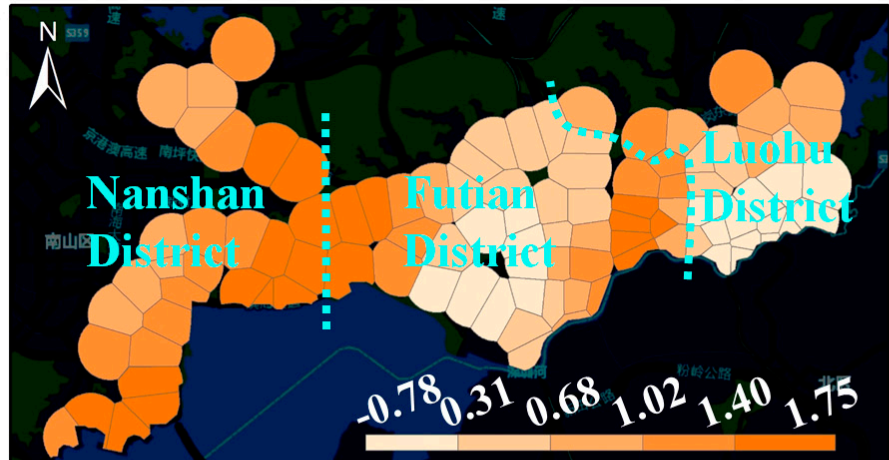

(a) Sed.Road on weekdays

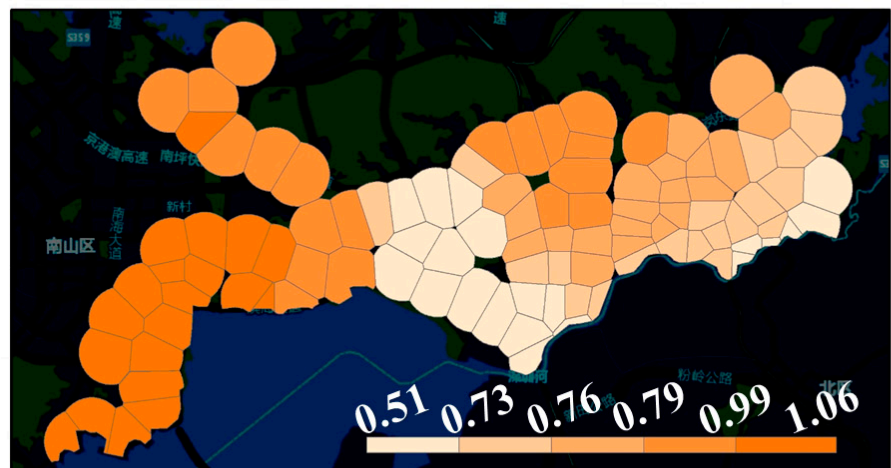

(b) Sed.Road on weekend

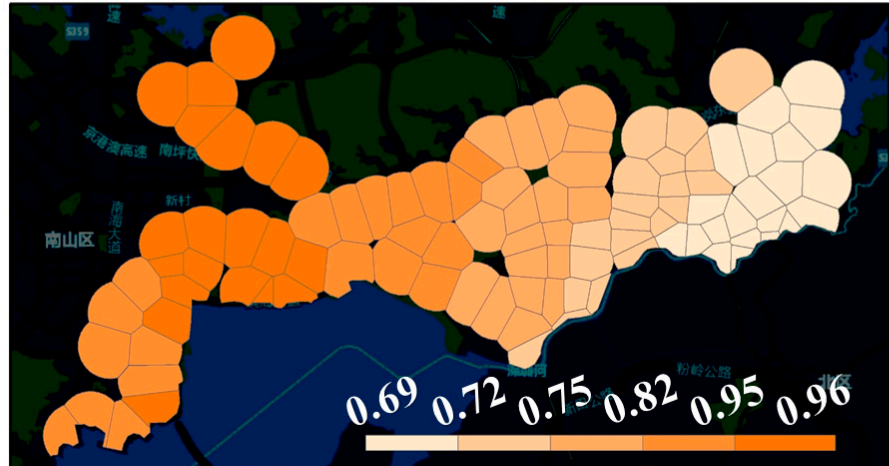

(c) Sed.Road on holidays

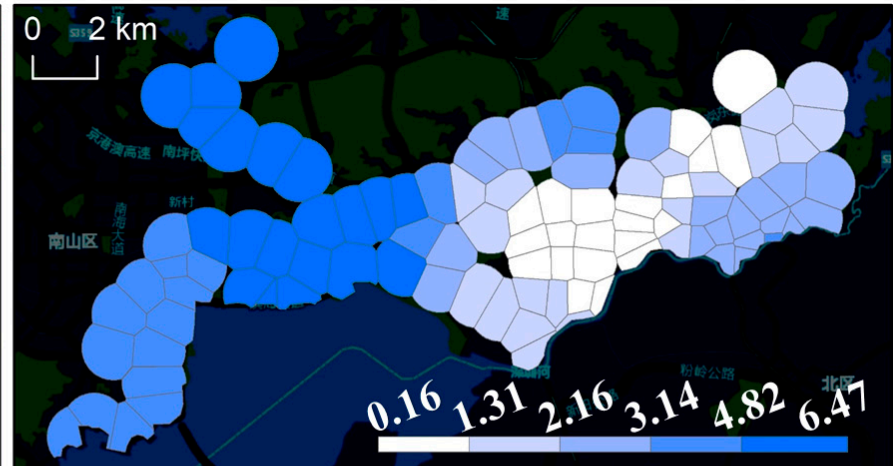

(d) Building on weekdays
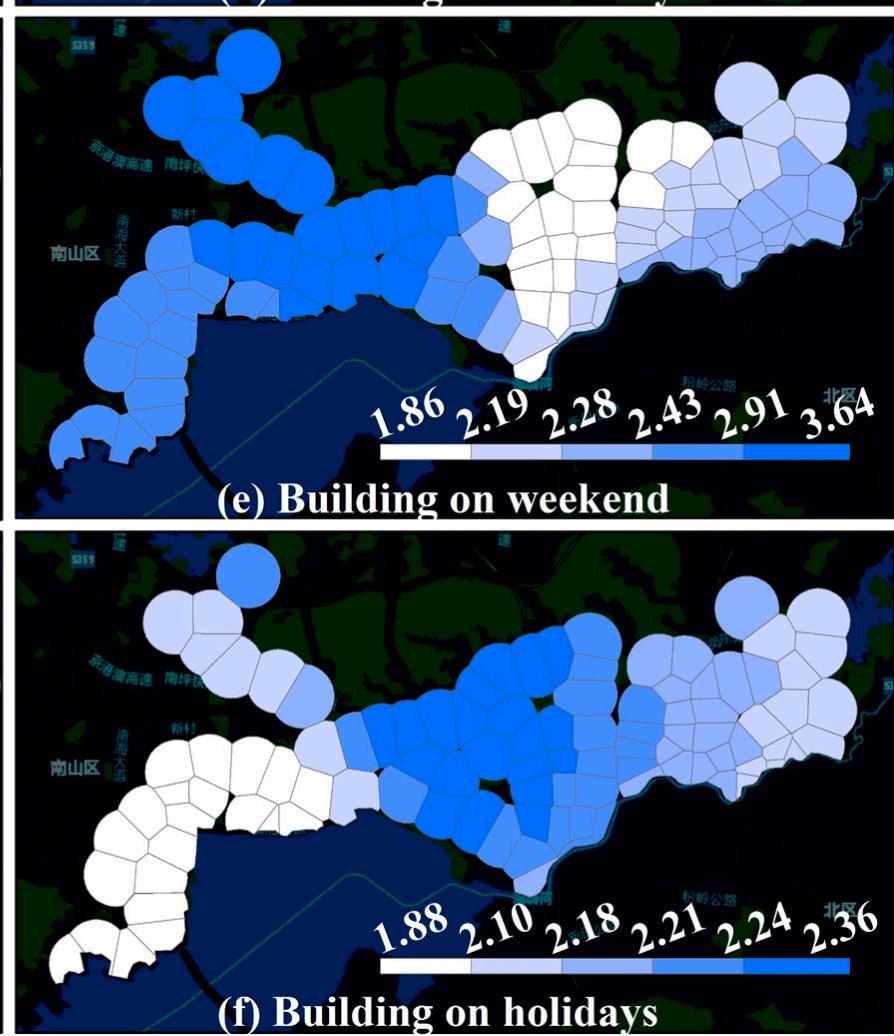

Figure 9. Local coefficients of secondary road density in GWR models of weekdays (a), weekend (b), and holidays (c). Local coefficients of building density in GWR models of weekdays (d), weekend (e), and holidays (f).

Figure 10 displays the local coefficients of floor area ratio, land use entropy, and realtime population density in GWR models on weekends and holidays. Generally, the floor area ratio was negatively associated with bike sharing usage, and land use entropy and real-time population density were positively related to bike usage. Although the floor area ratio was negatively associated with bike sharing usage in the study area, Futian district and southern Nanshan district had the weakest negative associations. This indicates that the floor area ratio might not directly and positively contribute to bike sharing usage. 

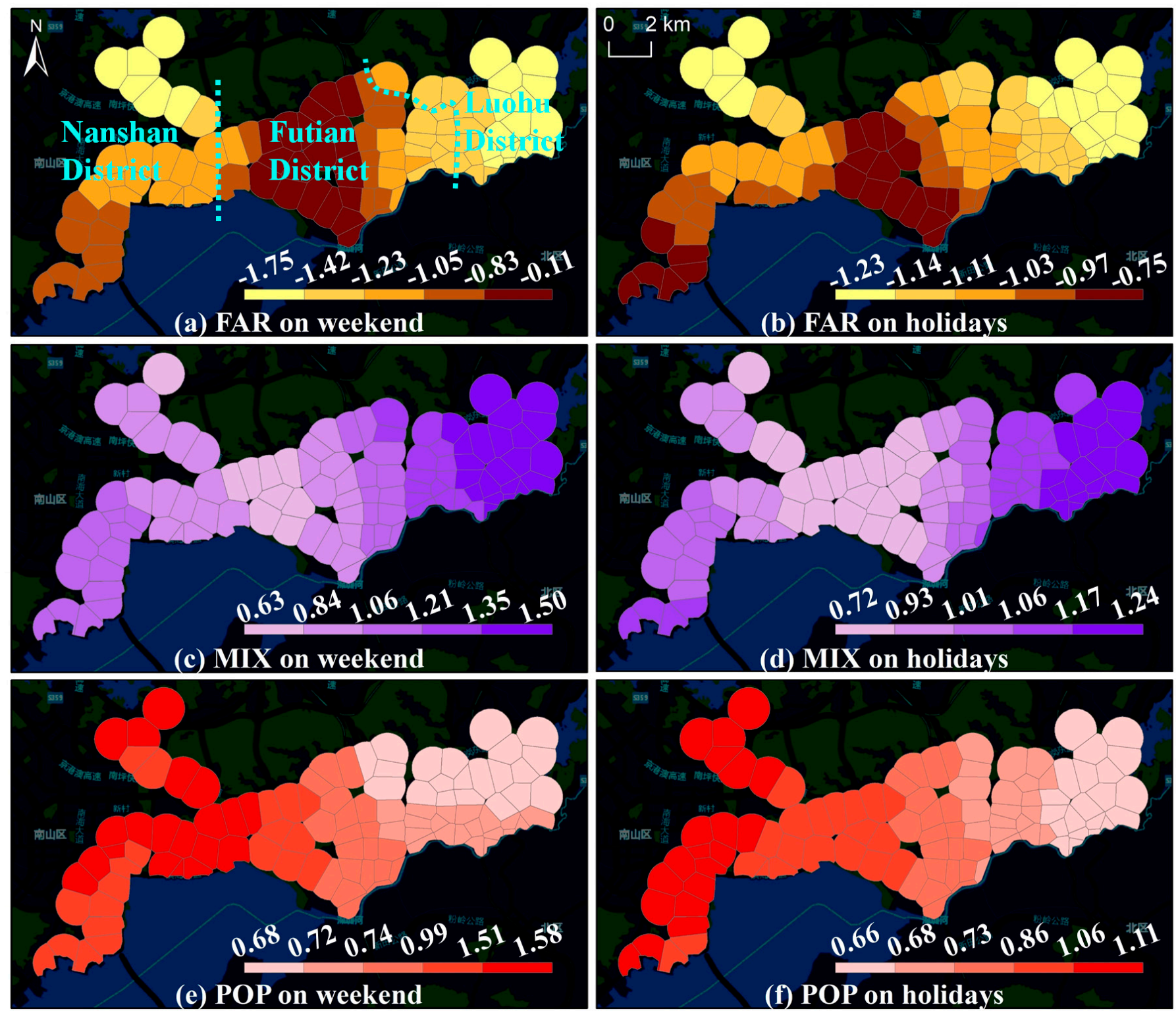

Figure 10. Local coefficients of floor area ratio (FAR) in GWR models of weekend (a) and holidays (b). Local coefficients of land use entropy (MIX) in GWR models of weekend (c) and holidays (d). Local coefficients of real-time population density (POP) in GWR models of weekend (e) and holidays (f).

Figure 10c,d show similar spatial distributions of land use entropy local coefficients on weekends and holidays. This suggests that the land use entropy in the eastern part of the study area (Luohu district) was stronger in association with bike sharing usage, and it was weak in Futian district. As areas with high land use mixtures tended to be multifunctional and diverse in both urban functions and travel purposes, it might be better able to attract or generate dockless bike sharing trips [2]. Therefore, the land use mixture in the eastern study area should be considered in promoting bike usage, especially in Luohu district.

Figure 10e,f show the local coefficients of real-time population density in GWR models on weekends and holidays, which were positively related to bike sharing usage globally. The local coefficients of POP of Nanshan district were the highest, followed by the Futian and Luohu district. This indicates that residents of Nanshan district are more reliant on the usage of dockless bike sharing for commuting than residents in Futian and Luohu districts. Therefore, areas with high local coefficients of POP are crucial in promoting bike sharing usage as they might directly represent the demand of local residents of bike sharing usage, which is consistent with previous studies [3-5]. 
Moreover, both bike usage on holidays and scenic spot distribution were first adopted in the association between bike sharing usage and built environment factors as explanatory and dependent variables, respectively. The results show that SCENIC was found to be significant only in the holidays model. Figure 11 shows the local coefficients of SCENIC in the GWR models of holidays. The scenic spot densities of the northern and middle Nanshan districts and northeastern Luohu district were strongly and positively associated with bike sharing usage on holidays. This indicates that, during holidays, areas with a high density of scenic spots might also attract or generate bike sharing trips; therefore, attention should also be paid to those areas to promote and manage dockless bike sharing usage on holidays.
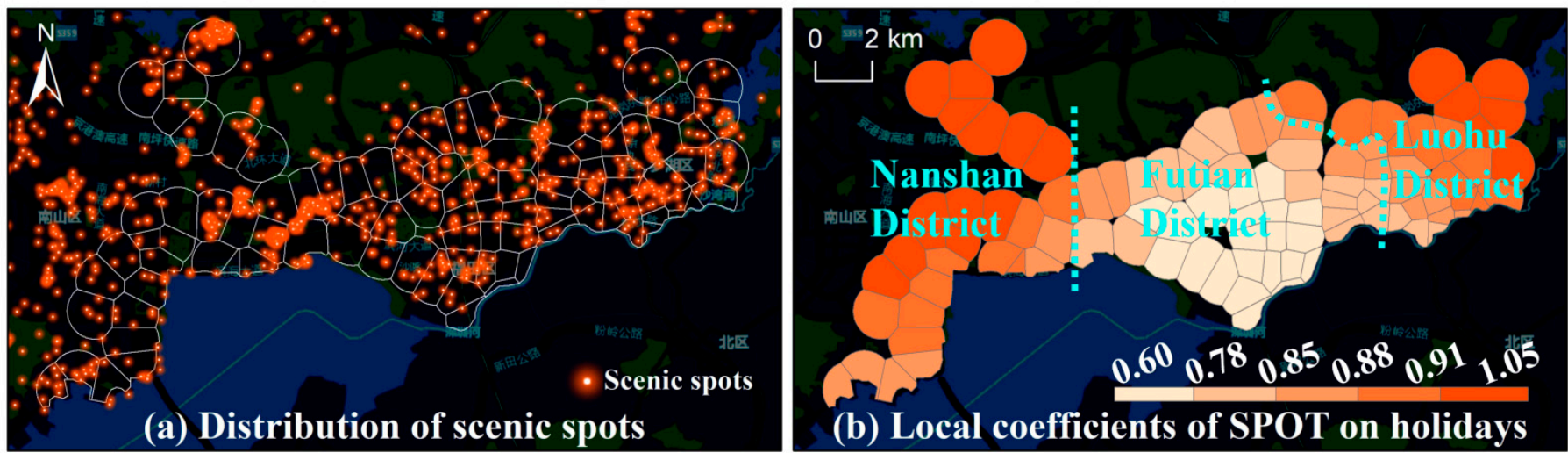

Figure 11. (a) Spatial distribution of scenic spots. (b) Local coefficients of SCENIC in GWR models of holidays.

\subsection{Planning Implications}

The above section presents the GWR model results in association between dockless bike sharing and urban greenness and other built environment factors, which is meaningful and efficient for related planning aimed at promoting bike sharing usage. The goal of this study was to understand the spatial association between urban greenness and other factors with bike sharing usage, and hence to put forward related suggestions and implications on how to promote the bike sharing usage effectively, specifically from a spatial perspective. It is important that areas with relatively low bike sharing usage are in need of suggestions on how to enhance bike usage. Areas with high local coefficients of factors in models tend to be more prospective and efficient in promoting bike sharing usage within them. Therefore, the two types of areas mentioned above will focus on planning implications.

In this study, planning implications were proposed based on the GWR results corresponding to weekdays, weekends, and holidays considering urban greenness and other built environment factors.

\subsubsection{Implications on Urban Greenness}

This study has proven that eye-level greenness positively contributes to dockless bike sharing usage compared with overhead view greenness. Therefore, the related planning implications focus on eye-level greenness.

On weekdays, the eastern part of Nanshan, western Futian (especially the junctions of them), and northeastern Futian and Luohu districts had low bike sharing usage (Figure 5a) and need planning recommendations. Among them, northeastern and southern Futian deserve the greatest improvement in eye-level greenness, and the western part of Nanshan ranks second, given that those areas have higher local coefficients of GVI (Figure 8e). Therefore, attention paid to them might be more efficient and helpful in promoting bike sharing usage.

On weekends, suggestions on eye-level greenness improvement seem to be more straightforward because of the spatial distribution trend of GVI local coefficients (Figure 8c). 
Low usage distribution on weekends was similar to weekdays, but Luohu district and northeastern Futian district experienced lower usage. Taking both the potential for improvement by greenness and bike usage into consideration, it is suggested that all areas with low weekend usage in Futian and Luohu districts should be improved in terms of eye-level urban greenness.

On holidays, low usage distribution was also similar for both weekdays and weekends, but more areas with low usage were observed (Figure $5 \mathrm{c}$ ). The higher local coefficients of GVI were located mainly in the southern Futian district (Figure 8d). Hence, eye-level greenness should be well designed and improved in southern Futian and southeastern Nanshan to effectively promote local bike sharing usage.

\subsubsection{Implications for Other Built Environment Factors}

First of all, secondary road density was positively associated with bike usage on weekdays, weekends, and holidays, which reflects that most bike sharing travel was along secondary roads, rather than primary and branch roads. Therefore, on weekdays, planning of secondary roads and en-route cycling facilities in Nanshan, western and eastern Futian, and northern Futian districts should be well designed and built to better serve cyclists, and hence promote bike sharing usage. Moreover, attention should be paid to Nanshan and northwestern Futian and Luohu districts on weekend, and Nanshan and Luohu districts on holidays.

Moreover, a positive association between land use mixture and bike sharing usage was found on weekends and holidays. Generally, areas with higher land use mixtures have greater potential to be more attractive to residents' daily travel and, hence, may be more capable of attracting or generating dockless bike sharing usage [2]. Similarly, the local coefficients of MIX on weekends (Figure 10c) are consistent with that of holidays (Figure 10d). The results indicate that the land use mixture of Luohu district has the greatest potential and efficiency to promote bike sharing usage on weekends and holidays. Therefore, urban planning considering land use mixture should be focused on Luohu district to promote the use of bike sharing on weekends and holidays.

Furthermore, real-time population density (Tencent user density) was applied in this study to replace the registered residential population. It was found to be positively related to bike sharing usage on weekends and holidays. High local coefficients of POP were observed in the Nanshan and Futian districts, especially in Nanshan (Figure 10e,f). This suggests that residents living in areas with high local coefficients of POP might tend to be more reliant on bike sharing, that is, more use demand might be generated in those areas. Therefore, the rebalance strategy by bike sharing operators should be focused on Nanshan and Futian districts, especially around Nanshan CBD, which might be helpful in promoting both usage efficiency and users' experience in those areas.

Finally, the positive association between bike sharing usage and scenic spot density was found only on holidays in the study, which was seldom observed in previous studies. It was remarkable that the scenic spot density of Nanshan and Luohu districts had a positive impact on bike sharing usage on public holidays (Figure 11). According to the results, densifying tourist attractions, especially in northern and western Nanshan districts and northeastern Luohu district, might contribute to the enhancement of dockless bike sharing usage on holidays.

\section{Conclusions}

The aim of this study was to explore the spatially varying association between dockless bike sharing usage around metro stations and urban greenness indicators using the GWR model. We have contributed to the literature in several ways. Firstly, we extended the related studies by obtaining longer time series of dockless bike sharing usage record data covering weekdays, weekends, and holidays (7 day National Holidays in China). Apart from weekdays and weekends, holidays are also an essential period that needs to be explored and compared in the association between urban greenness and bike sharing 
usage, during which time residents might tend to be more available to cycle for recreational purposes rather than for commuting to work and overtime on weekends [2]. In this study, variations in the impacts of eye-level greenness and overhead view greenness were compared, and the temporal differences in their associations with dockless bike sharing were explored including weekdays, weekends, and holidays. Secondly, we explored the spatially varying impacts of urban greenness on bike sharing usage by applying GWR, overcoming the shortage of non-spatial global modeling in previous studies. Global modeling results can only provide global suggestions based on global regression results. GWR can reflect the spatial heterogeneity of the local association between bike usage and urban greenness, as well as other built environment factors. Specific suggestions for local location can be promoted, which is more meaningful and efficient for cycling-related urban planning and design.

In conclusion, the results found that eye-level greenness was positively associated with bike sharing usage on weekdays, weekends, and holidays. Overhead view greenness was found to be negatively related to bike usage on weekends and holidays, and insignificant on weekdays. Moreover, building density and secondary road density were found to be positively associated on weekdays, weekend, and holidays; floor area ratio was negatively related on weekends and holidays; and land use mixture and population were positively associated on weekends and holidays. Finally, scenic spot density was found to be significantly positively associated with bike usage only on holidays. The innovation of this study lies in the urban planning implications based on the GWR results. For example, suggestions for eye-level greenness improvement may vary across the study area during different days. On weekdays, northeastern and southern Futian deserve the greatest improvement in eye-level greenness, and the western part of Nanshan ranks second, given that those areas have higher local coefficients of GVI.

The results can help provide suggestions for urban planning for specific locations and understand the spatial heterogeneity of the impacts of urban greenness and other built environment factors on dockless bike sharing usage. Dockless bike sharing is of great importance in residents' daily travel, release of air pollution, and reduction of private vehicle use, as well as the encouragement of public transport. Therefore, attention should be paid by urban planners and designers to the current situation and future planning of urban greenness from an eye-level perspective, rather than traditional greenness measurements such as NDVI from an overhead angle.

Despite the merits of this study, we must acknowledge that some limitations should be addressed in future studies. First, temporal characteristics have not been considered in the study models; hence, advanced methods involving temporal information should be adopted to further explore the mechanism of bike usage, such as the geographically and temporally weighted regression. In addition, future work should be extended by incorporating social demographic characteristics (age, education, and so on) into modeling to gain a better understanding of residents' cycling behavior.

Author Contributions: Conceptualization, Feng Gao and Shaoying Li; methodology, Feng Gao; software, Feng Gao; validation, Feng Gao and Shaoying Li; formal analysis, Feng Gao; investigation, Zhipeng Lai and Ziling Tan; resources, Shaoying Li and Xiaoming Zhang; data curation, Zhangzhi Tan; writing - original draft preparation, Feng Gao; writing—review and editing, Feng Gao and Shaoying Li; visualization, Feng Gao and Xiaoming Zhang; supervision, Shaoying Li; project administration, Shaoying Li; funding acquisition, Shaoying Li. All authors have read and agreed to the published version of the manuscript.

Funding: This research was funded by the National Natural Science Foundation of China [Grant No. 41871290, 41401432], Guangzhou Science and technology project [Grant No. 201904010198], and the Guangdong Enterprise Key Laboratory for Urban Sensing, Monitoring, and Early Warning [2020B121202019].

Institutional Review Board Statement: Not applicable.

Informed Consent Statement: Not applicable. 
Data Availability Statement: Not applicable.

Conflicts of Interest: The authors declare no conflict of interest.

\section{References}

1. Li, W.; Wang, S.; Zhang, X.; Jia, Q.; Tian, Y. Understanding intra-urban human mobility through an exploratory spatiotemporal analysis of bike-sharing trajectories. Int. J. Geogr. Inf. Sci. 2020, 34, 2451-2474. [CrossRef]

2. Gao, F.; Li, S.; Tan, Z.; Wu, Z.; Zhang, X.; Huang, G.; Huang, Z. Understanding the modifiable areal unit problem in dockless bike sharing usage and exploring the interactive effects of built environment factors. Int. J. Geogr. Inf. Sci. 2021, 35, 1-21. [CrossRef]

3. Lu, Y.; Yang, Y.; Sun, G.; Gou, Z. Associations between overhead-view and eye-level urban greenness and cycling behaviors. Cities 2020, 88, 10-18. [CrossRef]

4. Wang, R.; Lu, Y.; Wu, X.; Liu, Y.; Yao, Y. Relationship between eye-level greenness and cycling frequency around metro stations in Shenzhen, China: A big data approach. Sustain. Cities Soc. 2020, 59, 102201. [CrossRef]

5. Chen, Y.; Chen, Y.; Tu, W.; Zeng, X. Is eye-level greening associated with the use of dockless shared bicycles? Urban For. Urban Green. 2020, 51, 126690. [CrossRef]

6. Oja, P.; Titze, S.; Bauman, A.; de Geus, B.; Krenn, P.; Reger-Nash, B. Health benefits of cycling: A systematic review. Scand. J. Med. Sci. Sports 2011, 21, 496-509. [CrossRef]

7. Maibach, E.; Steg, L.; Anable, J. Promoting physical activity and reducing climate change: Opportunities to replace short car trips with active transportation. Prev. Med. 2009, 49, 326-327. [CrossRef]

8. Poirier, P.; Giles, T.D.; Bray, G.A.; Hong, Y.; Stern, J.S.; Pi-Sunyer, F.X. Obesity and cardiovascular disease: Pathophysiology, evaluation, and effect of weight loss: An update of the 1997 American Heart Association Scientific Statement on Obesity and Heart Disease from the Obesity Committee of the Council on Nutrition, Physical Activity, and Metabolism. Circulation 2006, 113, 898-918. [CrossRef]

9. Mcpherson, K. Reducing the global prevalence of overweight and obesity. Lancet 2014, 384, 728-730. [CrossRef]

10. Kushi, L.H.; Doyle, C.; McCullough, M.; Rock, C.L.; Demark-Wahnefried, W.; Bandera, E.V.; Gapstur, S.; Patel, A.V.; Andrews, K.; Gansler, T.; et al. American Cancer Society Guidelines on nutrition and physical activity for cancer prevention: Reducing the risk of cancer with healthy food choices and physical activity. CA Cancer J. Clin. 2012, 62, 30-67. [CrossRef]

11. Lee, I.M.; Shiroma, E.J.; Lobelo, F.; Puska, P.; Blair, S.N.; Katzmarzyk, P.T. Impact of physical inactivity on the world's major non-communicable diseases. Lancet 2012, 380, 219-229. [CrossRef]

12. Rabl, A.; de Nazelle, A. Benefits of shift from car to active transport. Transp. Policy 2012, 19, 121-131. [CrossRef]

13. Fraser, S.D.; Lock, K. Cycling for transport and public health: A systematic review of the effect of the environment on cycling. Eur. J. Public Health 2011, 21, 738-743. [CrossRef]

14. Cao, Y.; Shen, D. Contribution of shared bikes to carbon dioxide emission reduction and the economy in Beijing. Sustain. Cities Soc. 2019, 51, 101749. [CrossRef]

15. Lin, J.J.; Zhao, P.; Takada, K.; Li, S.; Yai, T.; Chen, C.H. Built environment and public bike usage for metro access: A comparison of neighborhoods in Beijing, Taipei, and Tokyo. Transp. Res. Part D Transp. Environ. 2018, 63, 209-221. [CrossRef]

16. Zhao, P.; Li, S. Bicycle-metro integration in a growing city: The determinants of cycling as a transfer mode in metro station areas in Beijing. Transp. Res. Part A Policy Pract. 2018, 99, 46-60. [CrossRef]

17. Martens, K. The bicycle as a feedering mode: Experiences from three European countries. Transp. Res. Part D Transp. Environ. 2004, 9, 281-294. [CrossRef]

18. Bachand-Marleau, J.; Larsen, J.; El-Geneidy, A.M. Much-anticipated marriage of cycling and transit: How will it work? Transp. Res. Rec. 2011, 2247, 109-117. [CrossRef]

19. Krenn, P.J.; Oja, P.; Titze, S. Route choices of transport bicyclists: A comparison of actually used and shortest routes. Int. J. Behav. Nutr. Phys. Act. 2014, 11, 31. [CrossRef]

20. Mertens, L.; Compernolle, S.; Deforche, B.; Mackenbach, J.D.; Lakerveld, J.; Brug, J. Built environmental correlates of cycling for transport across Europe. Health Place 2017, 44, 35-42. [CrossRef]

21. Mertens, L.; Compernolle, S.; Gheysen, F.; Deforche, B.; Brug, J.; Mackenbach, J. Perceived environmental correlates of cycling for transport among adults in five regions of Europe. Obes. Rev. 2016, 17, 53-61. [CrossRef]

22. Zahabi, S.A.H.; Chang, A.; Miranda-Moreno, L.F.; Patterson, Z. Exploring the link between the neighborhood typologies, bicycle infrastructure and commuting cycling over time and the potential impact on commuter GHG emissions. Transp. Res. Part D Transp. Environ. 2016, 47, 89-103. [CrossRef]

23. Chen, P.; Zhou, J.; Sun, F. Built environment determinants of bicycle volume: A longitudinal analysis. J. Transp. Land Use 2017, 10, 655-674. [CrossRef]

24. Christiansen, L.B.; Cerin, E.; Badland, H.; Kerr, J.; Davey, R.; Troelsen, J. International comparisons of the associations between objective measures of the built environment and transport-related walking and cycling: IPEN adult study. J. Transp. Health 2016, 3, 467-478. [CrossRef]

25. Xiao, Y.; Lu, Y.; Guo, Y.; Yuan, Y. Estimating the willingness to pay for green space services in Shanghai: Implications for social equity in urban China. Urban For. Urban Green. 2017, 26, 95-103. [CrossRef]

26. Wolch, J.R.; Byrne, J.; Newell, J.P. Urban green space, public health, and environmental justice: The challenge of making cities 'just green enough'. Landsc. Urban Plan. 2014, 125, 234-244. [CrossRef] 
27. Lachowycz, K.; Jones, A.P. Greenspace and obesity: A systematic review of the evidence. Obes. Rev. 2011, 12, e183-e189. [CrossRef]

28. Cole-Hunter, T.; Donaire-Gonzalez, D.; Curto, A.; Ambros, A.; Valentin, A.; GarciaAymerich, J. Objective correlates and determinants of bicycle commuting propensity in an urban environment. Transp. Res. Part D Transp. Environ. 2015, 40, 132-143. [CrossRef]

29. Kerr, J.; Emond, J.A.; Badland, H.; Reis, R.; Sarmiento, O.; Carlson, J. Perceived neighborhood environmental attributes associated with walking and cycling for transport among adult residents of 17 cities in 12 countries: The IPEN study. Environ. Health Perspect. 2015, 124, 290-298. [CrossRef] [PubMed]

30. Lu, M.; An, K.; Hsu, S.C.; Zhu, R. Considering user behavior in free-floating bike sharing system design: A data-informed spatial agent-based model. Sustain. Cities Soc. 2019, 49, 101567. [CrossRef]

31. Porter, A.K.; Kohl, H.W., III; Pérez, A.; Reininger, B.; Pettee Gabriel, K.; Salvo, D. Bikeability: Assessing the Objectively Measured Environment in Relation to Recreation and Transportation Bicycling. Environ. Behav. 2019, 52, 861-894. [CrossRef]

32. Sun, Y.; Du, Y.; Wang, Y.; Zhuang, L. Examining associations of environmental characteristics with recreational cycling behaviour by street-level Strava data. Int. J. Environ. Res. Public Health 2017, 14, 644. [CrossRef]

33. Takano, T.; Nakamura, K.; Watanabe, M. Urban residential environments and senior citizens' longevity in megacity areas: The importance of walkable green spaces. J. Epidemiol. Community Health 2002, 56, 913-918. [CrossRef] [PubMed]

34. de Vries, S.; van Dillen, S.M.; Groenewegen, P.P.; Spreeuwenberg, P. Streetscape greenery and health: Stress, social cohesion and physical activity as mediators. Soc. Sci. Med. 2013, 94, 26-33. [CrossRef]

35. Helbich, M.; Yao, Y.; Liu, Y.; Zhang, J.; Liu, P.; Wang, R. Using deep learning to examine street view green and blue spaces and their associations with geriatric depression in Beijing, China. Environ. Int. 2019, 126, 107-117. [CrossRef] [PubMed]

36. van Renterghem, T.; Botteldooren, D. View on outdoor vegetation reduces noise annoyance for dwellers near busy roads. Landsc. Urban Plan. 2016, 148, 203-215. [CrossRef]

37. Wang, R.; Helbich, M.; Yao, Y.; Zhang, J.; Liu, P.; Yuana, Y. Urban greenery and mental wellbeing in adults: Cross-sectional mediation analyses on multiple pathways across different greenery measures. Environ. Res. 2019, 176, 108535. [CrossRef]

38. Jing, F.; Liu, L.; Zhou, S.; Song, J.; Wang, L.; Zhou, H.; Wang, Y.; Ma, R. Assessing the Impact of Street-View Greenery on Fear of Neighborhood Crime in Guangzhou, China. Int. J. Environ. Res. Public Health 2021, 18, 311. [CrossRef]

39. Li, S.; Zhuang, C.; Tan, Z.; Gao, F.; Lai, Z.; Wu, Z. Inferring the trip purposes and uncovering spatio-temporal activity patterns from dockless shared bike dataset in Shenzhen, China. J. Transp. Geogr. 2021, 91, 102974. [CrossRef]

40. Wang, R.; Liu, Y.; Lu, Y.; Zhang, J.; Liu, P.; Yao, Y. Perceptions of built environment and health outcomes for older Chinese in Beijing: A big data approach with street view images and deep learning technique. Comput. Environ. Urban Syst. 2019, 78, 101386. [CrossRef]

41. Chen, Y.; Liu, X.; Li, X.; Liu, X.; Yao, Y.; Hu, G.; Xu, X.; Pei, F. Delineating urban functional areas with building-level social media data: A dynamic time warping (DTW) distance based k-medoids method. Landsc. Urban Plan. 2017, 160, 48-60. [CrossRef]

42. Niu, N.; Liu, X.; Jin, H.; Ye, X.; Liu, Y.; Li, X. Integrating multi-source big data to infer building functions. Int. J. Geogr. Inf. Sci. 2017, 31, 1871-1890. [CrossRef]

43. Yao, Y.; Liu, X.; Li, X.; Zhang, J.; Liang, Z.; Mai, K. Mapping fine-scale population distributions at the building level by integrating multisource geospatial big data. Int. J. Geogr. Inf. Sci. 2017, 31, 1220-1244. [CrossRef]

44. Li, S.; Lyu, D.; Huang, G.; Zhang, X.; Gao, F.; Chen, Y.; Liu, X. Spatially varying impacts of built environment factors on rail transit ridership at station level: A case study in guangzhou, china. J. Transp. Geogr. 2017, 82, 102631. [CrossRef]

45. Chiou, Y.; Jou, R.; Yang, C. Factors affecting public transportation usage rate: Geographically weighted regression. Transp. Res. Part A Policy Pract. 2015, 78, 161-177. [CrossRef]

46. Fotheringham, A.S.; Charlton, M.E.; Brunsdon, C. Geographically weighted regression: A natural evolution of the expansion method for spatial data analysis. Environ. Plan. A 1998, 30, 1905-1927. [CrossRef]

47. Lewandowska-Gwarda, K.; Antczak, E. Urban Ageing in Europe-Spatiotemporal Analysis of Determinants. ISPRS Int. J. Geo Inf. 2020, 9, 413. [CrossRef]

48. Tobler, W. A computer movie simulating urban growth in the Detroit region. Econ. Geogr. 1970, 46, 234-240. [CrossRef]

49. Brunsdon, C.; Fotheringham, A.S.; Charlton, M. Geographically Weighted Regression: A Method for Exploring Spatial Nonstationarity. Geogr. Anal. 1996, 28, 281-298. [CrossRef]

50. Fotheringham, A.S.; Brunsdon, C.; Charlton, M. Geographically Weighted Regression: The Analysis of Spatially Varying Relationships; John Wiley \& Sons: Hoboken, NJ, USA, 2003.

51. Charlton, M.; Fotheringham, A.S. Geographically Weighted Regression. A Tutorial on Using GWR in ArcGIS9.3. Available online:

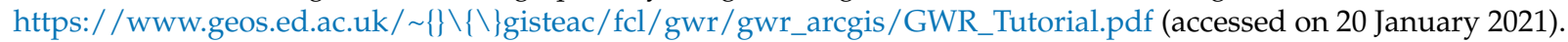

52. Zhou, X.; Yeh, A.G.O. Understanding the modifiable areal unit problem and identifying appropriate spatial unit in jobs-Housing balance and employment self-containment using big data. Transportation 2020, 1-17. [CrossRef]

53. Mitsuda, Y.; Yoshida, S.; Imada, M. Use of GIS-derived Environmental Factors in Predicting Site Indices in Japanese Larch Plantations in Hokkaido. J. For. Res. 2001, 6, 87-93. [CrossRef]

54. Li, S.; Lyu, D.; Liu, X.; Tan, Z.; Gao, F.; Huang, G.; Wu, Z. The varying patterns of rail transit ridership and their relationships with fine-scale built environment factors: Big data analytics from Guangzhou. Cities 2020, 99, 102580. [CrossRef] 
55. Yang, H.; Lu, X.; Cherry, C.; Liu, X.; Li, Y. Spatial variations in active model trip volume at intersections: A local analysis utilizing geographically weighted regression. J. Transp. Geogr. 2017, 64, 184-194. [CrossRef]

56. Li, B.; Cai, Z.; Jiang, L.; Su, S.; Huang, X. Exploring urban taxi ridership and local associated factors using GPS data and geographically weighted regression. Cities 2019, 87, 68-86. [CrossRef]

57. Silva, C.; Melo, S.; Santos, A.; Junior, P.A.; Sato, S.; Santiago, K.; Sá, L. Spatial Modeling for Homicide Rates Estimation in Pernambuco State-Brazil. ISPRS Int. J. Geo Inf. 2020, 9, 740. [CrossRef]

58. Shen, Y.; Zhang, X.; Zhao, J. Understanding the usages of dockless bike sharing in Singapore. Int. J. Sustain. Transp. 2018, 12, 686-700. [CrossRef]

59. Mooney, S.J.; Hosford, K.; Howe, B.; Yan, A.; Winters, M.; Bassok, A. Freedom from the station: Spatial equity in access to dockless bike share. J. Transp. Geogr. 2019, 74, 91-96. [CrossRef]

60. Nickkar, A.; Banerjee, S.; Chavis, C.; Bhuyan, I.A.; Barnes, P. A spatial-temporal gender and land use analysis of bikeshare ridership: The case study of Baltimore city. City Cult. Soc. 2019, 18, 100291. [CrossRef]

61. Bhuyan, I.A.; Chavis, C.; Nickkar, A.; Barnes, P. GIS-Based Equity Gap Analysis: Case Study of Baltimore Bike Share Program. Urban Sci. 2019, 3, 42. [CrossRef]

62. Xu, Y.; Chen, D.C.; Zhang, X.H.; Tu, W.; Chen, Y.Y.; Shen, Y.; Ratti, C. Unravel the landscape and pulses of cycling activities from a dockless bike-sharing system. Comput. Environ. Urban Syst. 2019, 75, 184-203. [CrossRef]

63. Zhu, R.; Zhang, X.; Kondor, D.; Santi, P.; Ratti, C. Understanding spatio-temporal heterogeneity of bike-sharing and scootersharing mobility. Comput. Environ. Urban Syst. 2020, 81, 101483. [CrossRef]

64. Feltynowski, M.; Kronenberg, J.; Bergier, T.; Kabisch, N.; Łaszkiewicz, E.; Strohbach, M.W. Challenges of urban green space management in the face of using inadequate data. Urban For. Urban Green. 2018, 31, 56-66. [CrossRef]

65. Łaszkiewicz, E.; Czembrowski, P.; Kronenberg, J. Creating a Map of the Social Functions of Urban Green Spaces in a City with Poor Availability of Spatial Data: A Sociotope for Lodz. Land 2020, 9, 183. [CrossRef]

66. Khalaim, O.; Zabarna, O.; Kazantsev, T.; Panas, I.; Polishchuk, O. Urban Green Infrastructure Inventory as a Key Prerequisite to Sustainable Cities in Ukraine under Extreme Heat Events. Sustainability 2021, 13, 2470. [CrossRef] 